\title{
Protected cat states from kinetic driving of a boson gas
}

\author{
G. Pieplow, C. E. Creffield $\odot$, and F. Sols \\ Departamento de Física de Materiales, Universidad Complutense de Madrid, E-28040 Madrid, Spain
}

(Received 14 June 2019; published 9 October 2019)

\begin{abstract}
We investigate the behavior of a one-dimensional Bose-Hubbard gas in both a ring and a hard-wall box, whose kinetic energy is made to oscillate with zero time average, which suppresses first-order particle hopping. For intermediate and large driving amplitudes, the system in the ring has similarities to the Richardson model, but with a peculiar type of pairing and an attractive interaction in momentum space. This analogy permits an understanding of some key features of the interacting boson problem. The ground state is a macroscopic quantum superposition, or cat state, of two many-body states characterized by the preferential occupation of opposite momentum eigenstates. Interactions give rise to a reduction (or modified depletion) cloud that is common to both macroscopically distinct states. Symmetry arguments permit a precise identification of the two orthonormal macroscopic many-body branches which combine to yield the ground state. In the ring, the system is sensitive to variations of the effective flux but in such a way that the macroscopic superposition is preserved. We discuss other physical aspects that contribute to protect the catlike nature of the ground state.
\end{abstract}

DOI: 10.1103/PhysRevResearch.1.033013

\section{INTRODUCTION}

The existence of macroscopic quantum superposition (MQS) states, or cat states, has long been one of the most counterintuitive predictions of quantum mechanics [1], as it is at odds with our daily perception of reality, where such states are not observed. The collapse of the Schrödinger cat state into one of its branches prevents us from directly observing coherent superpositions of macroscopically distinct states. This collapse is understood to be induced by the decohering effect of a dissipative environment which, in particular, can be a measuring apparatus [2-5]. Decoherence tends to act faster the larger the quantum system is [4].

Cat states may be viewed as a particular and extreme form of entanglement, in which various subsystems (at least some of them containing many particles) of a macroscopic system share their fate by highly correlating their response to specific questions asked in the form of a quantum measurement. So perhaps the most ubiquitous type of cat state is that formed in a measurement process where a microscopic quantum system becomes strongly correlated with the measuring apparatus. However, this type of MQS state is almost universally very fragile because, due to the macroscopic and noisy nature of the apparatus, the compound system quickly decays into one of the possible branches. For a given experimental run, we always observe the macroscopic apparatus in a well-defined configuration, from which we infer the state in which the quantum system is left. ${ }^{1}$

\footnotetext{
${ }^{1}$ This description applies literally only to the ideal measurement of a discrete quantum variable. We do not consider other types of measurement in the present discussion.

Published by the American Physical Society under the terms of the Creative Commons Attribution 4.0 International license. Further distribution of this work must maintain attribution to the author(s) and the published article's title, journal citation, and DOI.
}

Cat states are of great fundamental interest. For instance, they have been proposed to test macroscopic realism [6-10], which posits an observer-independent description of macroscopic objects whilst keeping the laws of quantum mechanics intact at the microlevel [9]. In that context, the characterization of an MQS state is especially relevant. Cat states also promise to be relevant for practical applications such as atom interferometry beyond the Heisenberg limit [11], precision measurements [12], quantum information tasks [13], and quantum metrology [14].

Quite generally, cat states are difficult to realize because coupling to the environment quickly destroys the coherent superposition of macroscopic states $[4,5,15]$. So the quest for the realization of MQS states is mostly about finding means to overcome decoherence and to probe the coherence between the macroscopically distinct states before it is lost.

In this paper, we present a system with the remarkable property of possessing a cat-type ground state which is unusually protected from decay into one of its branches. Following our previous work [16], in which we introduced the concept of "kinetic driving," where a system's kinetic energy is made to oscillate periodically in time with zero time average, we investigate the behavior of a one-dimensional interacting boson system, with both periodic and hard-wall boundary conditions. In each case, the ground state is a cat state involving two branches in which two different nonzero momentum states are macroscopically occupied.

In both the ring and the hard-wall cases, the system's resilience against collapse shows important differences as compared with other setups hosting catlike states. Most importantly, the circular boson superfluid preserves the MQS when subject to a spurious flux. Similarly, the gas between hard walls is fundamentally unaltered by a velocity drift due to gauge invariance. Another important feature is that the two condensates share the depletion cloud (here labeled reduction cloud), which provides a degree of protection against atom 
losses. Other aspects of resilience are discussed later in the paper.

Thus our goal here is to study the detailed properties of the catlike ground state for a kinetically driven boson system in both a ring and a box, where atoms satisfy periodic and hard-wall boundary conditions, respectively.

This paper is arranged as follows. Section II is devoted to a review of the experimental literature on cat states and an overview of representative theoretical papers. In Sec. III, we briefly reprise the main results of kinetic driving found in Ref. [16]. In Sec. IV, we explain how seemingly natural attempts to find a guiding simple picture fail. Notably, the naive picture of a ground state consisting of a coherent superposition of two opposite-momentum condensates each traveling with its own depletion cloud yields nonsensical results. Section $\mathrm{V}$ is devoted to an illuminating toy model that correctly describes the strong driving limit and qualitatively applies to a wider range of driving amplitudes. The resulting model Hamiltonian contains a pairing interaction which is a particular case of the general Richardson-Gaudin pairing model studied in the context of nuclear physics and small grain superconductivity $[17,18]$. Besides that pairing we additionally have an attractive interaction in momentum space that favors the macroscopic occupation of a single momentum mode [19]. We find that the depletion cloud of the conventional, undriven Bose-Hubbard (BH) model, with its well-known pairing structure, is replaced here by a reduction cloud based on a novel type of pairing and shared by the two condensate branches.

In Sec. VI, we investigate the many-body structure of the cat branches in the ring case. We find that the they are considerably more complex than the exclusive occupation of a nonzero momentum state. The collective ground state involves many momentum Fock configurations and there is no clear criterion to assign each configuration to a given branch. Indeed some momentum configurations contribute to both branches. We use a symmetry argument to sharply define the two orthonormal branches of the catlike ground state. In Sec. VII, we perform a similar study for the hard-wall box, suitably switching between a stationary-wave and a truncated plane-wave representation. This section concludes with a brief discussion of the case of harmonic confinement, whose ground state shows a similar MQS state structure, which underlines the robustness of the physical effects unveiled here.

The atypical character of effective Hamiltonian yields an unconventional particle current operator that, in both the ring and the box scenarios, yields a vanishing expectation value (for zero effective flux) for the two stationary states and cat branches forming the ground doublet. In a time-of-flight experiment, however, the behavior of the two cat branches would be markedly different.

Section VIII is devoted to a discussion of how different figures of merit help to measure the quality of the cat state. The discussion includes a brief review of the various "cattiness" measures that have been proposed in the literature, and introduces a new figure of merit. In Sec. IX, we investigate how realistic driving protocols enable the realization of the catlike ground state. We find that the ground state is easier to reach in the ring case. Finally, in Sec. X, we discuss why this system is more resilient to collapse than most cat-state proposals. In particular, we consider the possibility of decay due to spurious rotations or velocity drifts, atom losses and diagonal impurities. We also contemplate thermal excitations and coupling to an environment as possible causes of collapse.

After a concluding section, the paper is complemented by Appendices dealing with a variational calculation and a cat measure estimate.

\section{CAT STATES}

A cat state can be generally defined as the coherent superposition of two or a few macroscopically distinct states. Although the precise definition of "macroscopic" and "distinct" is by itself a subject of debate (as briefly reviewed in Sec. VIII for two branches), there is a considerable variety of physical systems for which cat states have been proposed and even realized.

Continuous superpositions of macroscopically distinct states (such as those appearing for double condensates [20,21]) may be considered MQS states, but usually are not referred to as cat states. The collapse of such states involves the measurement of a continuous macroscopic variable, which requires a specific discussion [22] that we shall not address here. A double condensate system may evolve alternating between cat states and continuous MQS states [21].

Early experiments prepared atoms in a correlated superposition of spatially separated [23] or internal [24] states. Evidence of MQS states was also found in persistent current states in superconducting quantum interference devices (SQUIDs) [25,26] following earlier experimental work on macroscopic quantum behavior in those systems [27-29], or in related Cooper pair boxes [30].

Diffraction, a characteristic quantum wave phenomenon, was observed in molecules as heavy as $\mathrm{C}_{60}$ [31] and even in larger molecules [32-34]. In the context of magnetism, coherent transitions between macroscopically distinct states involving different magnetic orientations of large molecules were observed [35-37].

Entanglement of up to four particles in trapped ion systems was detected in Ref. [38]. In similar systems, the number of entangled particles has later been increased to six [13] and fourteen [39]. Photonic systems have been tailored to yield small cat states $[40,41]$ in the optical and also in the microwave range $[42,43]$. In the optical range, these states have been increased to involve hundreds [44] or even hundreds of millions [45] of photons. In the microwave range, cats of up to hundreds of photons have been produced [46]. Cat states have also been realized in micromechanical oscillators [47], sometimes involving optical levitation of submicron particles [48]. In the context of cold bosonic atoms, a double-well spinor cat was realized [49].

On the theory side, early studies focused on superconducting devices $[6,50]$, including the coupling to a micromechanical resonator [51]. Other optomechanical oscillators have been proposed [52-55]. It has been argued that even a mirror in a cavity can be prepared in a cat state [56]. Optical systems are also good candidates for MQS based on the superposition of coherent states with very different amplitudes [57]. With the assistance of cavity collisions, few-atom systems can exhibit 
cat states of the Greenberger-Horne-Zeilinger (GHZ) type [58].

However, perhaps the physical system that has collected the largest group of cat state proposals is the atom BoseEinstein condensate [59-73], which is not surprising given its high tunability. To classify the various proposals, we may pay attention to the spatial distribution of the macroscopically distinct states, and the observable whose expectation value is different for the two branches. The most common choice is that of two (or a few) spatially separated atom clouds with the cat branches differing in the position of those clouds $[59,60,63,66,72,74]$. Cat states may also exist with a similar spatial distribution but with the relative phase playing the role of the macroscopic observable distinguishing the two branches [62,65,73]. The branches may occupy the same region of space but populating modes which differ in the density profile [75-77]. Another often-studied cat setup is that in which the two branches extend over the same ring but display different phase profiles, thus yielding distinct superfluid currents [68-71]. For quantum gases extended over a ring, but described by the attractive $\mathrm{BH}$ model, one may have all the atoms concentrated in a given site but hopping coherently between different sites in a highly correlated manner [64]. The atom spin can also be the observable distinguishing the two cat branches [78], sometimes correlated with the condensate position [61].

We may also compare the various guiding principles that have been proposed to reach a cat state once it has been identified. When the MQS state is the ground state of a given system, simple cooling to that ground state is very difficult because of the tiny energy splitting that separates the ground and first excited states [79]. Other approaches must thus be followed which can be roughly classified in three main categories: (i) projective measurement, (ii) dynamic evolution, and (iii) adiabatic preparation.

Examples in the first category are provided in Refs. [65,80], where the creation of macroscopic superpositions of atoms by some form of measurement on a BoseEinstein condensate was proposed. The setup usually involves a system in a continuous superposition of macroscopically different states that is subject to a limited measurement unable to resolve the difference between two distinct eigenvalues of the measured observable. A similar situation was found in Ref. [62] for the relative phase of two interfering condensates.

The proposals in Refs. [11,57,66,71,72] can be framed in the second category. Dynamic preparation means that an initial state is prepared and made to evolve in such a way that sometime during the evolution the system is known to be in a cat state. A variant of the proposal of Ref. [57] was realized experimentally in a photonic system [42]. The preparation of the initial state can be achieved by a sudden parameter change, for instance, the flip of the sign of the interaction in a bosonic atom gas [66], or a sudden change of the tunneling phase [71].

Adiabatic preparation (third method) has also been proposed to produce cat states [59,66]. The authors of Ref. [59] considered cooling to a ground state that is not catlike but which can be adiabatically transformed into a cat state. Adiabatic preparation was implemented in the experiment of Ref. [26]. There, a superposition of two macroscopically different flux states was created by slowly driving a SQUID towards a level anti-crossing. For superfluid systems, a general discussion of the difficulties to prepare MQS states in a rotating ring is presented in Ref. [69].

Finally, we may refer to the mechanisms that destroy the MQS state. The main cause of the fast decay of a Schrödinger cat state is the collapse into one of its branches due to decoherence [3]. The detailed mechanism is very sensitive to the particular physical realization but, as already stated, it is a general trend that the larger and more different the superposed branches are, the faster the collapse is. Particle losses are known to be an important source of decoherence for MQS states in ultracold atoms [59] or photonic systems [81]. Interaction of the condensate with the depletion cloud plays an important role in destroying the MQS of boson condensates in two immiscible internal states [61]. The authors of Ref. [66] identify the trapping lasers and the thermal cloud of a trapped condensate as the main cause of the loss of quantum coherence. Interestingly, some physical effects tend to inhibit decoherence. For instance, inhomogeneities in a ring [71] or interactions in a double well [72] can considerably delay the decay of the cat state. A discussion of the robustness of the more general concept of fragmented states, of which cat states are a subset, can be found in Ref. [82]

The unusual resilience to collapse is precisely one of the most interesting features of the setups considered in this paper. Section X contains a discussion of the possible mechanisms that may induce the decay of the cat state.

\section{KINETIC DRIVING IN THE RING}

In a previous paper [16], we considered a kinetically driven one-dimensional Bose-Hubbard $(\mathrm{BH})$ model with $L$ sites and periodic boundary conditions, i.e., a ring. We chose a specific time periodic hopping amplitude (hence kinetic driving), which made our starting point the Hamiltonian

$$
\begin{aligned}
\mathcal{H}(t)= & -J \cos (\omega t) \sum_{x=0}^{L-1}\left(a_{x}^{\dagger} a_{x+1}+a_{x+1}^{\dagger} a_{x}\right) \\
& +\frac{U}{2} \sum_{x=0}^{L-1} n_{x}\left(n_{x}-1\right),
\end{aligned}
$$

where $a_{x}, a_{x}^{\dagger}$ are the usual bosonic annihilation(creation) operators and $n_{x}=a_{x}^{\dagger} a_{x}$ is the number operator. The Hubbard interaction energy is given by $U>0$, and $J \cos (\omega t)$ is the time periodic tunneling amplitude between neighboring sites. We set $\hbar=1$, and measure momenta in units of the inverse lattice spacing. In Ref. [16], we derived an effective static model in the limit of high-frequency driving. The interested reader is referred to the Appendix of [16] for details of the derivation. In the (quasi-)momentum representation, the effective Hamiltonian for the driven system (1) is

$$
\begin{aligned}
H_{\kappa}= & \frac{U}{2 L} \sum_{l, m, n, p=0}^{L-1} \mathcal{J}_{0}\left[2 \kappa F\left(k_{l}, k_{m}, k_{n}, k_{p}\right)\right] \\
& \times a_{k_{p}}^{\dagger} a_{k_{n}}^{\dagger} a_{k_{m}} a_{k_{l}} \delta_{k_{l}+k_{m}, k_{n}+k_{p}},
\end{aligned}
$$


where

$$
F\left(k_{l}, k_{m}, k_{n}, k_{p}\right) \equiv \cos \left(k_{l}\right)+\cos \left(k_{m}\right)-\cos \left(k_{n}\right)-\cos \left(k_{p}\right),
$$

the Kronecker delta expresses momentum conservation mod $2 \pi, \mathcal{J}_{0}$ is the zeroth-order Bessel function, and the momenta are given by

$$
k_{p} \equiv 2 \pi p / L,
$$

with $p$ taking $L$ integer values from 0 to $L-1$, and $\kappa \equiv J / \omega$ is the driving parameter. In the ring $k_{p}$ is physically equivalent to $k_{p}+2 \pi n$ with $n \in \mathbb{Z}$. The properties of the system are determined by $\kappa$ together with the system size $L$ and the total particle number $N$. We choose $N$ to be even. The interaction $U$ becomes a global scaling factor and is the only remaining energy scale. The transformation relating the momentum and position representations is

$$
a_{k_{p}}=\frac{1}{\sqrt{L}} \sum_{x=0}^{L-1} e^{\mathrm{i} k_{p} x} a_{x}, \quad a_{x}=\frac{1}{\sqrt{L}} \sum_{p=0}^{L-1} e^{-\mathrm{i} k_{p} x} a_{k_{p}} .
$$

Next we briefly recapitulate some relevant results obtained in Ref. [16]. We found that the effective Hamiltonian (2) has a quantum phase transition of the Kosterlitz-Thouless type. Starting from a Mott-insulating state at $\kappa=0$ the system undergoes a transition at $\kappa \simeq 0.48$ into a fragmented superfluid state, which is (quasi)condensed into the momentum eigenstates of nonzero momenta $\pm \pi / 2$. The condensation is signaled by both the onset of clearly separated peaks in the momentum density $\left\langle n_{k}\right\rangle$ at $k= \pm \pi / 2,{ }^{2}$ and the closing of the Mott gap. Additional confirmation is obtained by measuring the Luttinger liquid parameter, $K_{b}$, which takes the universal value $K_{b}=1 / 2$ at the Kosterlitz-Thouless transition point.

Further information can be extracted from the two-particle momentum density. We found that the momentum-momentum correlation $\left\langle n_{k} n_{k^{\prime}}\right\rangle$ develops well separated peaks at $\left(k, k^{\prime}\right)=$ $\pm(\pi / 2, \pi / 2)$. Remarkably, the correlation is much smaller at $(\pi / 2,-\pi / 2)$, which leads us to conclude that the ground state is a Schrödinger catlike superposition of two macroscopically occupied momentum eigenstates, rather than something similar to a mere product state of $N / 2$ particles in $\pi / 2$, and $N / 2$ in $-\pi / 2$.

In Ref. [16], we provided some semiquantitative arguments to understand why the $\pm \pi / 2$ states are macroscopically occupied. In this paper, we present additional considerations that give us a deeper understanding of the special role played here by the momenta $\pm \pi / 2$. We may already point out that, if we take the expectation value of (2) in a many-body state consisting of all particles occupying the $k$ momentum eigenstate, the resulting energy is independent of $k$. This is a first clear indication that interactions play a crucial role in determining the structure of the ground state.

In the standard (undriven) Bose-Hubbard model, the occupation of the zero momentum state is favored because it

\footnotetext{
${ }^{2}$ We adopt the convention of using the subindex $p$ (or equivalent) for the momentum $k_{p}$ [as in Eq. (5)] only when deemed necessary; otherwise, we plainly refer to $k$ or $k^{\prime}$ with their sum understood as in the second Eq. (5).
}

minimizes the kinetic energy invested in the coherent hopping between sites. Deep in the superfluid regime the interactions may only represent an energetically small perturbation to this state, but one that is essential to achieve superfluidity. Here the situation is more involved because $H_{\kappa}$ only consists of interactions, and does not permit single-particle nearestneighbor hopping.

At this point we may also note some important symmetries. One can verify that $H_{\kappa}$ remains invariant under the transformations $k \rightarrow-k$ (time inversion) and $k \rightarrow \pm \pi+k$. This highlights the importance of the momentum eigenstates with momentum eigenvalues $0, \pi$, and $\pm \pi / 2$, since the unordered pairs $(0, \pi)$ and $(\pi / 2,-\pi / 2)$ remain invariant under the mentioned symmetry transformations. Thus the two components of each of those pairs must play symmetrical roles. In particular, if the ground state wave function contains a term where $\pi / 2$ is macroscopically occupied, then there must be another term where $-\pi / 2$ has exactly that occupation.

Later in the paper (see Sec. V and Appendix A), we will further argue why the macroscopic occupation of momenta $\pm \pi / 2$ can be expected to be energetically favored.

\section{TWO-MODE MODEL AND FAILURE OF THE BOGOLIUBOV APPROXIMATION}

One can gain more analytical insight by assuming the presence of only two modes $a \equiv a_{-\pi / 2}, b \equiv a_{\pi / 2}$. Then the Hamiltonian (2) becomes

$$
H_{2 \mathrm{LS}}=\frac{U}{2 L}\left(2 N^{2}-N-n_{a}^{2}-n_{b}^{2}+a^{\dagger^{2}} b^{2}+b^{\dagger^{2}} a^{2}\right),
$$

where we have used $n_{a}+n_{b}=N$, and the subindex 2LS stands for two-level system.

The above Hamiltonian can be diagonalized with the canonical transformation, $c=\frac{1}{\sqrt{2}}(a+b), \quad d=\frac{1}{\sqrt{2}}(a-b)$, which leads to

$$
H_{2 \mathrm{LS}}=\frac{U}{2 L}\left(2 N^{2}-2 N-4 n_{c} n_{d}\right),
$$

the normalized ground state being

$$
|\Psi\rangle=\frac{1}{(N / 2) !} \frac{1}{2^{N / 2}}\left(a^{\dagger^{2}}-b^{\dagger^{2}}\right)^{N / 2}|\mathrm{vac}\rangle .
$$

This state does not have the catlike momentum-momentum correlations that we observe numerically, and which ideally would be represented by a many-body state of the type

$$
(2 N !)^{-1 / 2}\left[\left(a^{\dagger}\right)^{N} \pm\left(b^{\dagger}\right)^{N}\right]|\mathrm{vac}\rangle \text {. }
$$

Actually, it can be shown that in the large- $N$ limit (8) satisfies $\left\langle n_{a} n_{b}\right\rangle /\left\langle n_{a} n_{a}\right\rangle \rightarrow 1 / 3$, which is much larger than the numerical result $\left\langle n_{a} n_{b}\right\rangle /\left\langle n_{a} n_{a}\right\rangle \ll 1$, while the same ratio is exactly zero for the state (9). The reason for this discrepancy lies in the coefficients accompanying the "center" configuration $\left|(N / 2)_{a},(N / 2)_{b}\right\rangle$ when the binomial expansion is implemented in Eq. (8). ${ }^{3}$ Their weights are simply too large. The failure of the two-mode approximation to reproduce the

\footnotetext{
${ }^{3}$ We introduce the notation $\left|M_{a}\right\rangle \equiv(M !)^{-1 / 2}\left(a^{\dagger}\right)^{M} \mid$ vac $\rangle$ whereby $\left|M_{a}\right\rangle$ is a state where $M$ particles occupy mode $a$.
} 


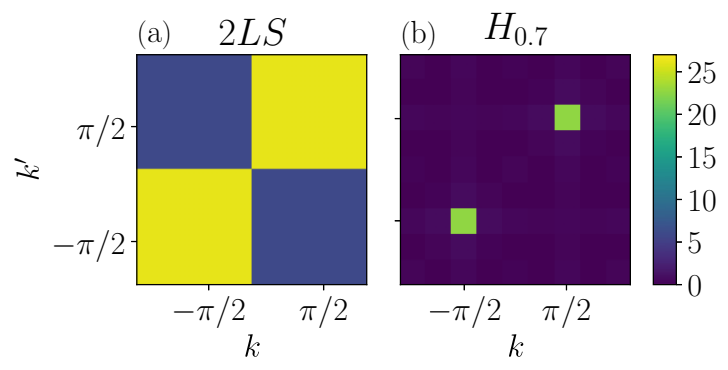

FIG. 1. Momentum correlations for (a) the two-mode approximation and (b) for the full effective Hamiltonian at $\kappa=0.7$. The respective ratios $\left\langle n_{\pi / 2} n_{\pi / 2}\right\rangle /\left\langle n_{-\pi / 2} n_{\pi / 2}\right\rangle$ are 4.3 and 45.5 , which means that the ground state of the two-mode approximation is much less catlike than that of the full model.

correct correlations further highlights the need to include the other modes $(k \neq \pm \pi / 2)$ and the interactions that connect them. In the next section, we address this question more precisely through a simplified description of the superfluid regime.

We note that, once we have decided to focus on the macroscopic occupation of two modes, (8) is the closest analog here to the Gross-Pitaevskii (GP) solution of the undriven BoseHubbard model, where only the occupation of one mode is considered. The GP approximation assumes a fully occupied one-atom mode of the type

$$
(N !)^{-1 / 2}\left(a^{\dagger}\right)^{N}|\mathrm{vac}\rangle
$$

One might be tempted to follow the steps of the undriven Bose gas and replace the operators $a$ and $b$ in Eqs. (2) and (6) by $c$ numbers. However, such an approximation is valid only when all relevant configurations have a large occupation of both modes $a$ and $b$, but we find numerically that this is not the case here. That approximation does apply to a state of the type (8) [this is clearer when (8) is written in terms of the $c, d$ operators] but, as already noted, the numerical results reveal that the system is far from that configuration. Most importantly, the weight of configurations in which both modes $( \pm \pi / 2)$ are largely occupied is tiny. Specifically, we know from numerical inspection that $\left\langle n_{k} n_{k^{\prime}}\right\rangle$ is very small away from $\left(k, k^{\prime}\right)= \pm(\pi / 2, \pi / 2)$, as can be appreciated in right panel of Fig. 1.

On the other hand, the macroscopic occupation of $\pm \pi / 2$ invites us to consider the picture of two condensates traveling in opposite directions, each one carrying its own depletion cloud. One might then perform a Bogoliubov calculation based on the macroscopic occupation of one of the two preferred momentum states, hoping that a meaningful physical picture will be obtained. However, such a simple-minded Bogoliubov calculation yields anomalous results, the main one being that all quasiparticles have zero energy. Thus it becomes clear that the equivalent of the depletion cloud for the fragmented, catlike condensate has to be qualitatively different from the conventional case. We will refer to the set of momentum-representation Fock states with at least one momentum $k \neq \pm \pi / 2$ occupied as the reduction cloud, to clearly distinguish it from the conceptually simpler depletion cloud of the undriven Bose-Hubbard model. In the next section,

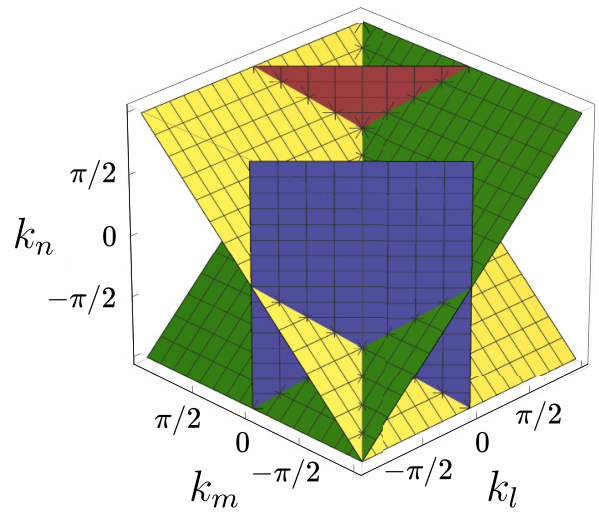

FIG. 2. The four planes (each plotted with a different color) containing the scattering events whose amplitude in $H_{\kappa}$ is independent of $\kappa$. We only show the first Brillouin zone in $k_{l}, k_{m}, k_{n}$ space.

we study an approximate Hamiltonian that provides valuable insights on the structure of the reduction cloud.

\section{LARGE $\kappa$ LIMIT: TOY MODEL}

As we have just argued, we need to fundamentally depart from the Bogoliubov-de Gennes approach, and turn to other approximation schemes. In order to investigate the structure of the reduction cloud, we resort to a simplified Hamiltonian and study it in the superfluid regime. We will assume that, in Eq. (2), the only relevant scattering processes are those for which the argument of the Bessel function is zero. This approximation becomes exact in the the limit $\kappa \rightarrow \infty$.

For those elementary processes, the matrix element is independent of $\kappa$. They satisfy the condition

$$
F\left(k_{l}, k_{m}, k_{n}, k_{p}\right)=0,
$$

i.e., $\mathcal{J}_{0}(2 \kappa F)=1$ in Eq. (2). By invoking momentum conservation, (11) becomes

$$
\cos \left(k_{l}\right)+\cos \left(k_{m}\right)-\cos \left(k_{m}+k_{l}-k_{n}\right)-\cos \left(k_{n}\right)=0 .
$$

This is true provided any of the following four conditions is fulfilled:

$$
k_{l}=k_{n}, \quad k_{m}=k_{n}, \quad k_{l}+k_{m}= \pm \pi,
$$

the first two describing the absence of a collision. These three equations can be neatly viewed as four planes in the first Brillouin zone of $\left(k_{l}, k_{m}, k_{n}\right)$ space. This is illustrated in Fig. 2, where the third Eq. (13) appears unfolded into two planes plotted in the same color. Any point on these four planes represents a scattering event that has amplitude $U / 2 L$. Since $\lim _{x \rightarrow 0} \mathcal{J}_{0}(x)=1$, these are the amplitudes that dominate in the large- $\kappa$ limit. In that limit, $H_{\kappa}$ effectively becomes

$$
H_{\infty}=\frac{U}{2 L}\left(2 N^{2}-N-\sum_{k} n_{k}^{2}+\sum_{k \neq k^{\prime} k+k^{\prime} \neq \pi} a_{\pi-k}^{\dagger} a_{k}^{\dagger} a_{\pi-k^{\prime}} a_{k^{\prime}}\right) .
$$

Hereafter the sums over momenta $k, k^{\prime}$ are understood in the sense of (4) and (5). 


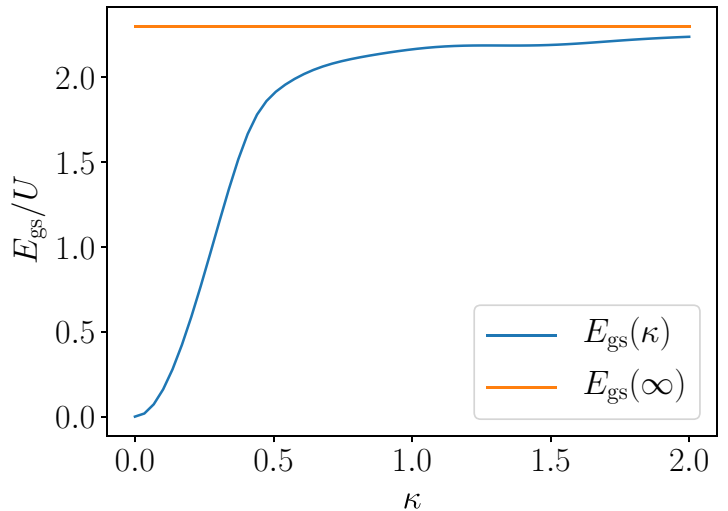

FIG. 3. Comparison of the ground state energy of $H_{\kappa}$ and $H_{\infty}$ for eight particles on eight sites. As expected, for increasing $\kappa$ the ground state energy of $H_{\kappa}$ approaches that of the asymptotic model. We have checked this convergence for multiple system sizes and fillings.

Although this Hamiltonian is exact only for $\kappa \rightarrow \infty$, we will see that it adequately captures many of the properties of our system for moderately large values of $\kappa$. Figures 3 and 4 a show that, even for $\kappa \lesssim 1$, the ground state energy and the momentum density of $\tilde{H}_{\kappa}$ are very close to those of $H_{\infty}$. Comparison of the right Fig. 1 and the lower Fig. 4 shows that

(a)

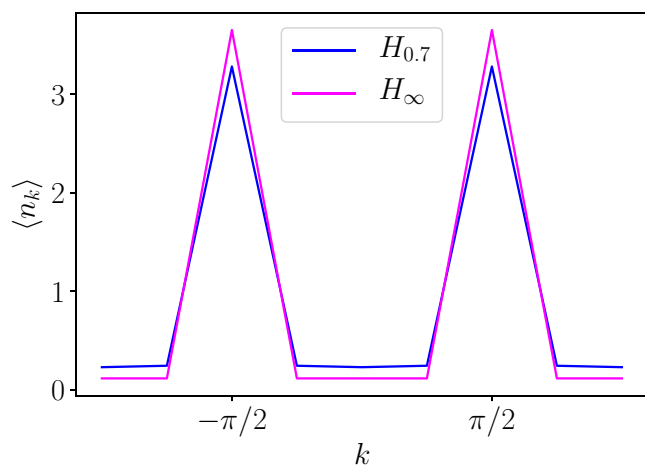

(b)

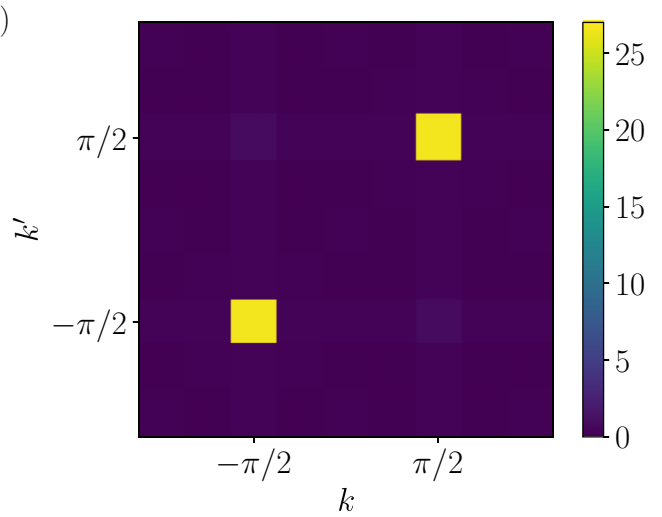

FIG. 4. (a) Momentum density $\left\langle n_{k}\right\rangle$ for the ground state of $H_{0.7}$ and $H_{\infty}$ for eight particles on eight sites. Both show clearly separated peaks around $k= \pm \pi / 2$. (b) momentum-momentum correlations for the ground state of $H_{\infty}$. Again, they are clearly peaked around $\pm \pi / 2$ and show negligible cross correlations, indicating a catlike state rather than a product state. Note that the case $\kappa=0.7$ is plotted in right Fig. 1. the two-particle momentum densities are also very similar. This justifies the detailed study of $H_{\infty}$ as a reference model for the more physical parameter regime of moderate $\kappa$.

By removing the scaling factor $U / 2 L$, and the terms that are constant for a given $N$, we trim $H_{\infty}$ down to the simple toy model

$$
h_{\infty}=-\sum_{k} n_{k}^{2}+\sum_{\substack{k \neq k^{\prime} \\ k+k^{\prime} \neq \pi}} a_{\pi-k}^{\dagger} a_{k}^{\dagger} a_{\pi-k^{\prime}} a_{k^{\prime}}
$$

which adequately captures, for a broad range of $\kappa$ values $(\kappa \sim$ 0.5 and larger), the properties of the true ground state in the superfluid sector.

\section{A. Connection to the Richardson model}

The toy model (15) has interesting connections to other areas of physics. The second term shows a pairing-type interaction in which only collisions between particle pairs with total momentum $\pi$ are allowed.

Pairing interactions (albeit typically between pairs of zero total momentum) appear in the theory of superconductivity and in nuclear physics contexts. This topic has a rich history in which many exactly solvable models have been developed and studied. An excellent review on this topic can be found in Ref. [18], where frequent references are made to the work of Richardson [17], who found a numerically exact solution for what has later become known as the Richardson model. The theory was rediscovered and successfully applied to mesososcopic superconducting metallic grains [83,84]. Since then a whole class of integrable so-called Richardson-Gaudin models have been found [18]. Despite $h_{\infty}$ not being included in that set of models, we can make use of many of the tools developed in Ref. [17], and the forthcoming analysis is an adaption of elements of Richardson's theory to our model.

The main difference between the Richardson model and $h_{\infty}$ is the first term on the r.h.s. of Eq. (15), which, as noted in Ref. [19], may be viewed as an attractive interaction in momentum space. A particle pair in our system is created by the operator $B_{k}^{\dagger}=a_{\pi-k}^{\dagger} a_{k}^{\dagger}$. Just as in Ref. [17], we can define the seniority operator

$$
v_{k}=\left|n_{k}-n_{\pi-k}\right|,
$$

which counts the number of unpaired particles with momentum $k$ or $\pi-k .^{4}$ We note that

$$
\left[h_{\infty}, v_{k}\right]=0,
$$

and define the seniority of an eigenstate of $h_{\infty}$ as its eigenvalue with respect to the operator

$$
v=\frac{1}{2} \sum_{k} v_{k},
$$

which measures the total number of unpaired particles, the factor $1 / 2$ being introduced to prevent double counting.

The conservation of seniority $\left(\left[h_{\infty}, v\right]=0\right)$ permits a helpful block-diagonalization of $h_{\infty}$, since only configurations

\footnotetext{
${ }^{4}$ Actually in Ref. [17] seniority was defined in a formally different way because it was initially applied to fermions.
} 
with the same seniority are connected by $h_{\infty}$. In particular, states that do not contain any pairs $(v=N)$ are eigenstates of the pairing term with eigenvalue 0 and thus eigenstates of $h_{\infty}$. The states with the lowest energy within the class of $v=N$ are those of the type $\left|N_{k \neq \pm \pi / 2}\right\rangle$, their energy being $-N^{2}$, where

$$
\left|N_{q}\right\rangle \equiv(N !)^{-1 / 2}\left(a_{q}^{\dagger}\right)^{N}|\mathrm{vac}\rangle
$$

is a state with $N$ particles in momentum $q$.

An important sector in the block-diagonalized Hamiltonian is $v=0$, i.e., the set of states that are only made of configurations where all particles are paired. We refer to them as fully paired states. Within this set of configurations, only $\left|N_{ \pm \pi / 2}\right\rangle$ involve the exclusive occupation of a single one-atom state, since $\pm \pi / 2$ are the only two momenta that form a pair with themselves. Similarly to $\left|N_{k \neq \pm \pi / 2}\right\rangle$, they benefit the most from the "on-site" attractive interaction in momentum space. However, unlike $\left|N_{k \neq \pm \pi / 2}\right\rangle,\left|N_{ \pm \pi / 2}\right\rangle$ are not eigenstates of the repulsive pairing term in Eq. (15), because of the contribution

$$
\sum_{k \neq \pm \pi / 2} a_{\pi-k}^{\dagger} a_{k}^{\dagger}\left(a_{\pi / 2} a_{\pi / 2}+a_{-\pi / 2} a_{-\pi / 2}\right)+\text { H.c. }
$$

there included. Intuitively one expects that states involving only $\pi / 2$ or $-\pi / 2$ or both will further lower their energy by mixing with configurations which include pairs with $k \neq$ $\pm \pi / 2$. As announced in the previous section, we will refer to those states as the reduction cloud of the $\pm \pi / 2$ condensates.

The main differences between the reduction cloud and the conventional depletion cloud are that (i) the pairs of the reduction cloud have total momentum $\pi$ (in contrast to zero for the depletion cloud) and, most importantly, (ii) the reduction cloud is shared by the two condensates. The reason for the second difference is clear from (20): the creation of a pair $(k, \pi-k)$ of total momentum $\pi$ (with $k \neq \pm \pi / 2$ ) can borrow the momentum indistinguishably from condensate pairs $(\pi / 2, \pi / 2)$ or $(-\pi / 2,-\pi / 2)$, which have the same total crystal momentum, namely, $\pi$.

Indeed it can be proven by a variational calculation that mixing $\left|N_{ \pm \pi / 2}\right\rangle$ with other fully paired (but containing at least one $k \neq \pm \pi / 2$ ) further lowers the energy beyond $-N^{2}$. The details of this can be found in Appendix A. This result confirms the intuition that the two states $\left|N_{ \pm \pi / 2}\right\rangle$ benefit energetically from the mixing with the interaction-induced reduction cloud. More specifically, $\left|N_{ \pm \pi / 2}\right\rangle$ mixes with other fully paired states of the type $\left|(N-2)_{ \pm \pi / 2}, 1_{k}, 1_{\pi-k}\right\rangle$ with $k \neq \pm \pi / 2$, which lowers the energy thanks to the pairing interactions in $H_{\kappa}$. By contrast, states of the type $\left|N_{k}\right\rangle$ with $k \neq \pm \pi / 2$ do not have this possibility, since the pairing term does not allow them to mix with other configurations. As already noted, they are eigenstates of $H_{\kappa}$.

The block diagonalization greatly helps in reducing the numerical overhead when studying the ground state of the system. For $v=0$ and unit filling, one only has to deal with $\left(\begin{array}{c}N-1 \\ N / 2\end{array}\right)$ configurations instead of $\left(\begin{array}{c}2 N-1 \\ N\end{array}\right) .{ }^{5}$ For eight particles on eight sites this already makes a big difference. Instead

\footnotetext{
${ }^{5}$ Recall that $N$ bosons distributed in $L$ sites yield $\left(\begin{array}{c}N+L-1 \\ N\end{array}\right)$ distinct configurations.
}

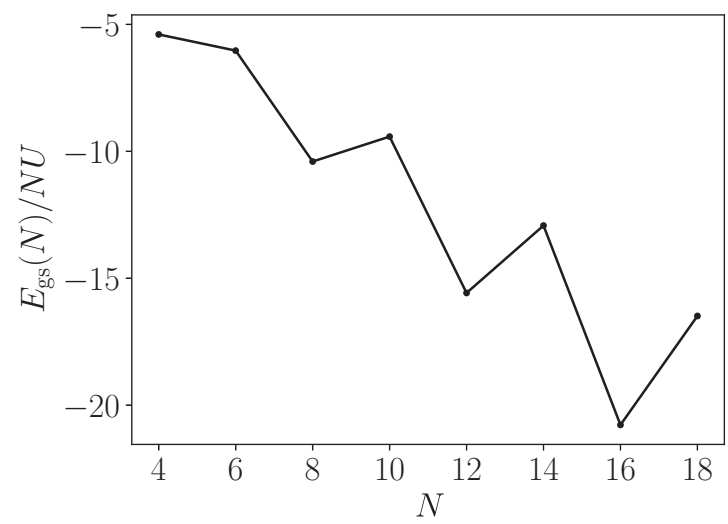

FIG. 5. Energy per particle of the ground state of $h_{\infty}$ for different system sizes with unit filling. $N=L$ is the number of sites in the ring. A system that does not include $\pm \pi / 2$ in its set of momenta has a slightly higher energy per particle than an adjacent system of similar size that does contain it. The latter case corresponds to $N / 4$ being an integer.

of 6435 configurations, one only has to work with 70 . This allows the system size to be increased up to 18 particles on 18 sites. In Fig. 5, we see an interesting scaling of the energy per particle when increasing the system size. The energy drops in a zig-zag pattern. This is connected to the fact that only when $\pm \pi / 2$ are among the allowed momenta can we have fully paired states involving the occupation of just two modes, namely, $\pm \pi / 2$. This is possible only when $N / 4$ is an integer.

\section{B. Ground state}

Inspection of $h_{\infty}$ shows that all the momenta $k \neq \pm \pi / 2$ play an equivalent role. This suggests that the ground state of $h_{\infty}$ can be written as a fully paired state of the form:

$$
\begin{aligned}
\left|\Psi_{0}\right\rangle= & \sum_{m=0}^{N / 2} \sum_{l=0}^{(N-2 m) / 2} C_{m, l}\left[\left|(N-2 m-2 l)_{-\frac{\pi}{2}},(2 l)_{\frac{\pi}{2}}\right\rangle\right. \\
& \left.+\left|(2 l)_{-\frac{\pi}{2}},(N-2 m-2 l)_{\frac{\pi}{2}}\right\rangle\right] \\
& \times \sum_{\sum n_{k}=m} Q_{\left\{n_{k}\right\}} \sum_{P}\left|P\left\{n_{k}\right\}\right\rangle,
\end{aligned}
$$

where the sum over $\left\{n_{k}\right\}$ runs over all possible occupation numbers $n_{k}$ of the pairs $(k, \pi-k)$, each one characterized by the momentum $k$ satisfying $0 \leqslant k<\pi / 2$, so that in each sequence $\left\{n_{k}\right\}$, a given number $n_{k}$ represents the two-mode state $\left|n_{k}, n_{\pi-k}\right\rangle$ containing $n$ particles in momentum $k$ and $n$ particles in momentum $\pi-k$. We also sum over all possible permutations $P$ of the sequence of pair occupation numbers. Each permutation $P$ acting on $\left\{n_{k}\right\}$ yields the same number sequence but distributed throughout the set of state pairs (always with $k \neq \pm \pi / 2$ ) in a different way. The resulting pair configuration is represented by $\left|P\left\{n_{k}\right\}\right\rangle$ and must have the same weight as $\left|\left\{n_{k}\right\}\right\rangle$. Thus the ansatz (21) is expected to be exact within the toy model, something which we have confirmed numerically.

To further understand the above state we may write down the leading contributions to the ground state. If $Q_{m}$ is defined as $Q_{\left\{n_{k}\right\}}$ for the particular sequence in which $m$ particle pairs 
transferred to the reduction cloud are concentrated in one mode pair [as usual labeled $(k, \pi-k)$ with $k \neq \pm \pi / 2$ ], then we can write

$$
\begin{aligned}
\left|\Psi_{0}\right\rangle= & C_{0,0} Q_{0}\left(\left|N_{-\frac{\pi}{2}}, 0_{\frac{\pi}{2}}\right\rangle+\left|0_{-\frac{\pi}{2}}, N_{\frac{\pi}{2}}\right\rangle\right) \\
& +C_{1,0} Q_{1}\left(\left|(N-2)_{-\frac{\pi}{2}}, 0_{\frac{\pi}{2}}\right\rangle\right. \\
& \left.+\left|0_{-\frac{\pi}{2}},(N-2)_{\frac{\pi}{2}}\right\rangle\right) \sum_{k \neq \pm \pi / 2}\left|1_{k}, 1_{\pi-k}\right\rangle \\
& +C_{0,1} Q_{0}\left(\left|(N-2)_{-\frac{\pi}{2}}, 2_{\frac{\pi}{2}}\right\rangle+\left|2_{-\frac{\pi}{2}},(N-2)_{\frac{\pi}{2}}\right\rangle\right) \\
& \left.\left.+C_{1,0} Q_{2}(\mid N-4)_{-\frac{\pi}{2}}, 0_{\frac{\pi}{2}}\right\rangle+\left|0_{-\frac{\pi}{2}},(N-4)_{\frac{\pi}{2}}\right\rangle\right) \\
& \times \sum_{k \neq \pm \pi / 2}\left|2_{k}, 2_{\pi-k}\right\rangle+\cdots,
\end{aligned}
$$

where the terms are written in order of decreasing value of $\left|C_{m, l} Q_{m}\right|^{2}$, as obtained numerically for the case of eight particles on eight sites. 6

The expansion (22) has some interesting information. The first term is clearly identifiable as the ideal Schrödinger catlike superposition of the two macroscopically occupied orbitals $\pm \pi / 2$ (sometimes called a NOON state). The second term is the largest contribution to the reduction cloud. The third term represents an internal exchange of one pair between the two main configurations of the fragmented condensate, without intervention of the reduction cloud. The fourth term represents the exchange of four particles between the fragmented condensate and the reduction cloud all going to the same mode pair; and so on.

The many-body states (21) and (22) show very clearly that the reduction cloud is shared by the two branches (or macroscopically distinct configurations) of the cat state. This fact definitely eliminates the naive picture of the cat state formed by two macroscopic branches each carrying its own distinguishable depletion cloud. Some of these points are further discussed in Appendix A.

As we have noted, when a particle pair of total momentum $\pi$ is created, its momentum can be equivalently viewed as coming from either the $\pi / 2$ or the $-\pi / 2$ condensates. In practice this means that, as we see in Eqs. (21) and (22), such a pair (with $k \neq \pm \pi / 2$ ) factors out from a coherent, still catlike superposition of the two different macroscopic branches.

\section{First excited state}

Numerical inspection shows that the main difference between the ground and the first excited states is their behavior under time reversal, i.e., the transformation that changes the sign of all momenta $(k \rightarrow-k)$

$$
T a_{k} T^{\dagger}=a_{-k} .
$$

While the ground state is symmetric with regard to time inversion, the first excited state is antisymmetric. For the toy model, the first excited state is very similar to (21) and (22) except for

\footnotetext{
${ }^{6}$ Note that these decreasing coefficients are not necessarily correlated with the relative weight, within the many-body ground state, of a given type of configuration, since they are multiplying nonnormalized many-body states.
}

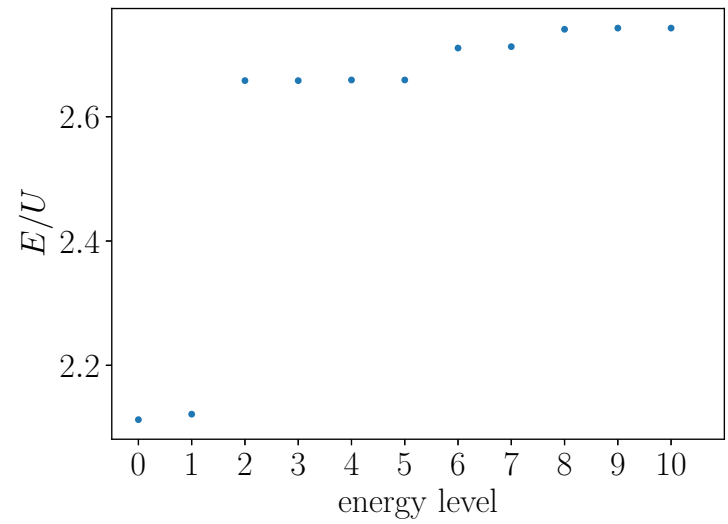

FIG. 6. Low-lying excitations of $H_{\kappa}$ with $\kappa=0.8$ for eight particles on a ring of eight sites. The lowest two energy levels form an almost degenerate doublet, separated by a large energy gap from the rest of the spectrum.

the relative sign between $\left|(N-2 m-2 l)_{-\pi / 2},(2 l)_{\pi / 2}\right\rangle$ and $\left|(2 l)_{-\pi / 2},(N-2 m-2 l)_{\pi / 2}\right\rangle$, which is flipped. As a consequence, the first excited state avoids all configurations for which $N-2 m-2 l=2 l$.

The energy spectrum for $H_{0.8}$ is shown in Fig. 6. The lowest-lying doublet (formed by the similar ground and first excited states) is clearly well-isolated from the higher-lying excited states. We also note that in general the values of $\left|C_{m, l}\right|$ for a given $m, l$ are different in the ground and the first excited state. This is correlated with the fact that the two lowestenergy states are not degenerate (see Fig. 6). Physically, this nondegeneracy is a subtle issue that we discuss in the next section.

\section{MANY-BODY PLANE WAVES}

Interestingly, we find numerically that the natural orbitals (defined as the eigenstates of the reduced one-particle density matrix) are just plane waves. Of them, the two most occupied ones have momenta $\pm \pi / 2$ for both the ground state and the first excited state. Moreover, as already noted, we find numerically that the ground state and the first excited state are, respectively, symmetric and antisymmetric under time reversal. Importantly, this results holds when all coefficients of the ground state wave function in the momentum Fock representation are real. These properties suggest another angle from which to view the relation between the ground state $\left|\Psi_{0}\right\rangle$ and the first excited state $\left|\Psi_{1}\right\rangle$, namely, as the symmetric and antisymmetric superposition of two collective plane waves with average momentum $\pm \pi / 2$. Specifically, we are led to the following approximate picture for the ground doublet:

$$
\begin{aligned}
& \left|\Psi_{0}\right\rangle \simeq|C(\pi / 2)\rangle, \\
& \left|\Psi_{1}\right\rangle \simeq \mathrm{i}|S(\pi / 2)\rangle,
\end{aligned}
$$

where

$$
\begin{aligned}
|C(k)\rangle & \equiv \frac{1}{\sqrt{2}}[|\Phi(k)\rangle+|\Phi(-k)\rangle], \\
|S(k)\rangle & \equiv \frac{-\mathrm{i}}{\sqrt{2}}[|\Phi(k)\rangle-|\Phi(-k)\rangle],
\end{aligned}
$$




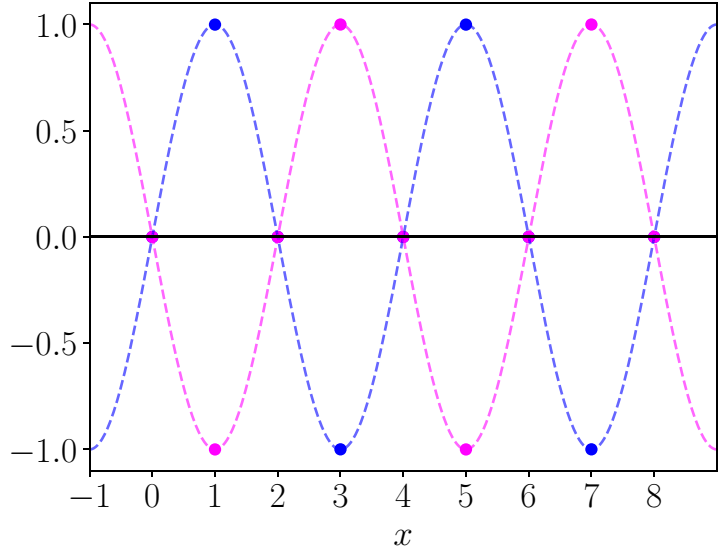

FIG. 7. Exact (dots) and ideal (dashed lines) natural orbitals in the ring. The real part of the wave function is plotted.

and $|\Phi(k)\rangle=\left|N_{k}\right\rangle$ is a many-body state with all particles in momentum $k$, its wave function being

$$
\left\langle x_{1}, \ldots, x_{N} \mid \Phi(k)\right\rangle=L^{-N / 2} \exp \left(\mathrm{i} k \sum_{i} x_{i}\right),
$$

where $x_{i}$ is the space coordinate of the $i-$ th boson. Here letters $C$ and $S$ are reminiscent of the $\sin (k x)$ and $\cos (k x)$ wave functions which these states acquire in the single-particle $(N=1)$ case. In this limit, $|\Phi(k)\rangle$ becomes the single-particle state of wave function $L^{-1 / 2} \exp (\mathrm{i} k x)$.

In Fig. 7, we plot the wave function of the numerical and ideal plane wave orbitals for eight particles on a ring of eight sites. The difference between the numerical and the ideal results is indistinguishable to the eye.

We can now use the approximate picture (24) and (25) as a guide to organize the numerical results.

Once we have identified the highly correlated nature of the macroscopic occupation of momenta $\pm \pi / 2$, as shown in Fig. 1(b) (which reproduces Fig. 5 of Ref. [16]), we may wonder whether we can neatly separate the two branches of the catlike state. If we were dealing with an ideal cat state of the type (9) as exemplified in Eq. (25), the answer would be easy: the two branches would be $|\Phi( \pm \pi / 2)\rangle$, both being states of the form (10).

The situation is more complicated when, instead of the ideal cat states (9) and (25), we have to deal with numerically obtained states which literally involve thousands of momentum configurations. Considering that all intervening Fock states have total momentum $0 \bmod 2 \pi$, we must rule out the possibility of establishing a criterion to decide to which branch a given momentum configuration contributes. This is even more so if (as will in fact be the case) a given Fock state may contribute to both cat branches.

Equations (24) and (25) offer a simple path to identify the two cat branches if $|C(\pi / 2)\rangle$ and $|S(\pi / 2)\rangle$ are replaced by the true ground and first excited states. One only has to invert (25) to propose

$$
\left|\Psi_{ \pm}\right\rangle=\frac{1}{\sqrt{2}}\left(\left|\Psi_{0}\right\rangle \pm\left|\Psi_{1}\right\rangle\right)
$$

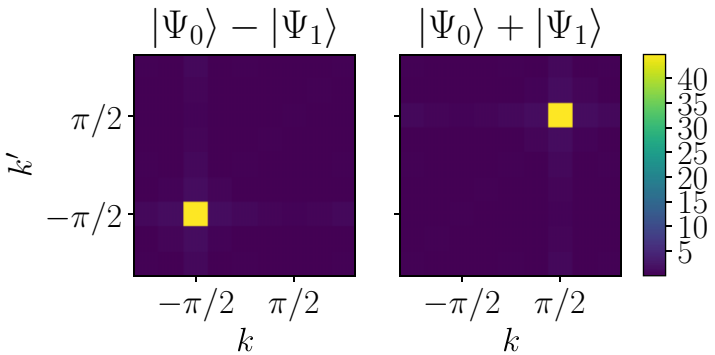

FIG. 8. Momentum correlations of the states $\left|\Psi_{ \pm}\right\rangle=\frac{1}{\sqrt{2}}\left(\left|\Psi_{0}\right\rangle \pm\right.$ $\left.\left|\Psi_{1}\right\rangle\right)$ constructed from the superposition of the ground and first excited states. The isolated peaks clearly suggest that the ground state is formed by two counter-propagating many-body configurations akin to collective plane waves. Here, $\kappa=0.7$.

and write

$$
\begin{aligned}
& \left|\Psi_{0}\right\rangle=\frac{1}{\sqrt{2}}\left(\left|\Psi_{+}\right\rangle+\left|\Psi_{-}\right\rangle\right), \\
& \left|\Psi_{1}\right\rangle=\frac{1}{\sqrt{2}}\left(\left|\Psi_{+}\right\rangle-\left|\Psi_{-}\right\rangle\right)
\end{aligned}
$$

with the orthogonality of $\left|\Psi_{0}\right\rangle$ and $\left|\Psi_{1}\right\rangle$ guaranteeing

$$
\left\langle\Psi_{+} \mid \Psi_{-}\right\rangle=0 .
$$

In Fig. 8, we show the two-particle momentum density of $\left|\Psi_{ \pm}\right\rangle$. The distinct single peaks at $\pm(\pi / 2, \pi / 2)$ clearly confirm the adequacy of the criterion (27) to cleanly construct the two cat branches. Despite its simple appearance, we emphasize that Fig. 8 shows $\left\langle n_{k} n_{k^{\prime}}\right\rangle$ for a numerically obtained state $\left|\Psi_{ \pm}\right\rangle$involving thousands of momentum configurations of which $\left|N_{ \pm \pi / 2}\right\rangle$ (with all particles in $\pm \pi / 2$ ) is only that with the largest weight. Specifically, for $N=L=8$, we find that the states $\left|N_{ \pm \pi / 2}\right\rangle$ add up to a normalization weight of approximately $50 \%$.

Remarkably, the decomposition in cat branches such as that shown in Eq. (28) with the results of Fig. 8 is also possible for a boson gas between hard walls, as we discuss in the next section.

Before shifting to the hard-wall case, we finish this section with a note on the nondegeneracy of the ground state doublet shown in Fig. 6. In the particular case of $N=1$, (24) yields two degenerate states, since $\cos (\pi x / 2)$ and $\sin (\pi x / 2)$ are wave functions connected by a symmetry operation, namely, a space translation of one lattice spacing. Interestingly, the same analysis for $N$ particles shows that $|C(\pi / 2)\rangle$ and $|S(\pi / 2)\rangle$ differ by a global $1 / N$ translation. For $N>1,1 / N$ is less than a lattice spacing and thus such a translation does not yield a degenerate state. However, practical degeneracy is obtained for $N \gg 1$. This argument, developed for noninteracting bosons, provides a semiquantitative explanation of the small but nonzero splitting of the interacting ground state doublet shown in Fig. 6. It also suggests that the splitting vanishes in the thermodynamic $(N \rightarrow \infty)$ limit.

\section{A. Particle current}

It is tempting to view the branches (27) as collective states where many particles "travel" with an average momentum of $\pm \pi / 2$. However, this picture is invalidated under closer 
inspection. The unusual character of the effective Hamiltonian (2) yields an also unconventional particle current operator $I_{K}$. If, as a result of a twist in the periodic boundary conditions, all allowed momenta are shifted by an amount $\theta$, the matrix elements in $H_{\kappa}$ change accordingly. The space-averaged particle current operator, $I_{\kappa}=L^{-1} \partial H_{\kappa} / \partial \theta$ can thus be written as

$$
\begin{aligned}
I_{\kappa}= & \frac{U \kappa}{L^{2}} \sum_{l, m, n, p=0}^{L-1} \mathcal{J}_{1}\left[2 \kappa F\left(k_{l}, k_{m}, k_{n}, k_{p}\right)\right] \\
& \times G\left(k_{l}, k_{m}, k_{n}, k_{p}\right) a_{k_{p}}^{\dagger} a_{k_{n}}^{\dagger} a_{k_{m}} a_{k_{l}} \delta_{k_{l}+k_{m}, k_{n}+k_{p}},
\end{aligned}
$$

where $\mathcal{J}_{1}$ is the first-order Bessel function and

$$
G\left(k_{l}, k_{m}, k_{n}, k_{p}\right) \equiv \sin \left(k_{l}\right)+\sin \left(k_{m}\right)-\sin \left(k_{n}\right)-\sin \left(k_{p}\right) .
$$

Clearly, the expectation value of $I_{\kappa}$ vanishes for a state of the type $|\Phi(k)\rangle=\left|N_{k}\right\rangle$ where all particles are in the same momentum $k$. Numerically, we confirm

$$
\left\langle\Psi_{ \pm}\left|I_{\kappa}\right| \Psi_{ \pm}\right\rangle=\left\langle\Psi_{0,1}\left|I_{\kappa}\right| \Psi_{0,1}\right\rangle=0 .
$$

However, despite this apparent lack of dynamics, the two branches $\left|\Psi_{ \pm}\right\rangle$will behave very differently in a time-of-flight experiment in which the crystal momentum in the lattice becomes the linear momentum in the vacuum as the confining optical lattice is switched off. Moreover, we find that, in the presence of a finite twist in the periodic boundary conditions, the ground state carries a nonzero current (not shown). Interestingly, the nonzero value of the current depends crucially on the presence of the reduction cloud.

\section{KINETIC DRIVING BETWEEN HARD WALLS}

Here we investigate the effect of kinetically driving a onedimensional boson system in the presence of hard walls. With respect to the flat ring scenario studied in Ref. [16] and in the previous sections, there are similarities but also some key differences.

The symmetry is greatly reduced and momentum conservation is lost due to lack of translational invariance. The momentum values $0, \pi$ do not play symmetric roles anymore, nor do in general the momenta $k, \pi-k$ with $k \neq \pi / 2$, as was the case for the ring. The natural orbitals are not plane waves. The toy model which, derived in the large- $\kappa$ limit, helped us understand much of the physics in a wide range of $\kappa$ values, does not work here anymore.

We no longer can use the plane wave expansion in Eq. (5) to derive the effective Hamiltonian. We must rather introduce stationary waves satisfying the hard-wall boundary conditions. The usual trick is to extend the lattice by two sites and use stationary waves to expand the creation and annihilation operators in position space [see, e.g., Ref. [85]]:

$$
\begin{aligned}
& a_{x}=\sqrt{\frac{2}{L+1}} \sum_{l=1}^{L} \sin \left(\tilde{k}_{l} x\right) a_{\tilde{k}_{l}}, \\
& a_{\tilde{k}_{l}}=\sqrt{\frac{2}{L+1}} \sum_{x=1}^{L} \sin \left(\tilde{k}_{l} x\right) a_{x} .
\end{aligned}
$$

Crucially, the wave vector $\tilde{k}_{l}$ is defined

$$
\tilde{k}_{l}=\pi l /(L+1),
$$

where $l$ takes $L$ integer values from 1 to $L$, so that $\tilde{k}_{l} \in$ $(0, \pi)$. This contrasts with the definition of $k_{l}$ given in Eq. (4) for plane waves in the ring, with $k_{l} \in[0,2 \pi)$. Here the momentum density of states is twice as high as for plane waves.

The effective Hamiltonian with hard-wall boundary conditions is therefore

$$
\begin{aligned}
H_{\kappa}^{\mathrm{hw}}= & \frac{2 U}{(N+1)^{2}} \sum_{x=1}^{N} \sum_{l, m, n, p=1}^{N} \sin \left(\tilde{k}_{l} x\right) \sin \left(\tilde{k}_{m} x\right) \sin \left(\tilde{k}_{n} x\right) \\
& \times \sin \left(\tilde{k}_{p} x\right) \mathcal{J}_{0}\left[2 \kappa F\left(\tilde{k}_{l}, \tilde{k}_{m}, \tilde{k}_{n}, \tilde{k}_{p}\right)\right] a_{\tilde{k}_{p}}^{\dagger} a_{\tilde{k}_{n}}^{\dagger} a_{\tilde{k}_{m}} a_{\tilde{k}_{l}},
\end{aligned}
$$

with $F$ defined in Eq. (3). Its derivation is completely analogous to that of $H_{\kappa}$, which was presented in Ref. [16]. Note that the main difference with respect to (2) is the loss of momentum conservation, and hence the necessary preservation of the the residual sum over the position $x$. If the mode functions were plane waves this would yield a simple Kronecker delta, as in Eq. (2).

The derivation of the toy model for the ring relied on the simple solutions to Eq. (12), which include momentum conservation. An analogous derivation for the hard-wall effective Hamiltonian is too involved to produce a similarly simple Hamiltonian for large $\kappa$. Indeed we find that some properties of the ground state such as its momentum density significantly change for $\kappa>0.8$ (not shown). By contrast, in the ring case the large- $\kappa$ limit remains consistently smooth for arbitrarily large values of $\kappa$.

As to the Luttinger liquid analysis which we made in Ref. [16] for the ring case, we note that the hard-wall boundary conditions drastically alter the superfluid correlations [86]. This makes it harder to reliably extract Luttinger parameters from small systems. Since the biggest system we can numerically investigate is eight particles on eight sites, we will not attempt here to explore the Luttinger liquid properties of the confined boson system.

\section{A. Plane-wave representation}

Although plane waves do not provide a natural basis for quantum particles between hard walls, it is nevertheless possible to introduce them through the transformation:

$$
a_{x}=\frac{1}{\sqrt{L}} \sum_{l=1}^{L} e^{\mathrm{i} k_{l} x} a_{k_{l}} .
$$

In this expansion, we implicitly set the plane waves to zero on the fictitious sites $x=0, L+1$ where the hard wall is supposed to be. Because of this we refer to (38) as a truncated plane wave expansion. This expansion is not useful in deriving the effective Hamiltonian, but will become important later in the physical representation of the results. 
The stationary plane waves can be transformed into truncated plane waves via

$$
a_{\tilde{k}_{l}}=\sqrt{\frac{2}{L(L+1)}} \sum_{m=1}^{L} \sum_{x=1}^{L} e^{\mathrm{i} k_{m} x} \sin \left(\tilde{k}_{l} x\right) a_{k_{m}}
$$

with $\tilde{k}_{l}$ and $k_{m}$ are defined as in Eqs. (36) and (4), respectively. Unlike in the continuum limit case, the discrete nature of the sum and the fundamental difference between the definitions of $\tilde{k}_{l}$ and $k_{m}$ make this transformation nontrivial. It is also the reason why expressing (37) in the plane wave representation does not provide any immediate advantage.

\section{B. Numerical results}

As for the case of periodic boundary conditions, we have studied the momentum density and the momentummomentum correlations. To properly compare the ring and hard-wall cases, we work in the truncated plane wave representation, which is perfectly reachable through the transformation (39). Once the ground state has been obtained in terms of stationary modes, it is easy to investigate the system in the truncated plane-wave representation. Interestingly, the properties stay largely intact as compared with the ring case. As $\kappa$ increases, two distinct peaks form in the momentum density and the momentum-momentum correlation also shows distinct peaks at $\pm(\pi / 2, \pi / 2)$ (shown in Fig. 9). Such correlated peaks indicate that the ground state remains Schrödinger catlike even for hard-wall boundary conditions. The ground state is a coherent superposition of two macroscopically distinct states, one with most atoms at positive momenta (centered around $\pi / 2$ ) and the other in the time-reversed configuration.

As in the ring case, we can make use of symmetry to better understand the catlike structure of the ground and first excited states. Space inversion around the midpoint of the chain amounts to the transformation

$$
I a_{\tilde{k}_{l}} I^{\dagger}=\left\{\begin{array}{c}
-a_{\tilde{k}_{l}} l \text { even } \\
a_{\tilde{k}_{l}} l \text { uneven }
\end{array},\right.
$$

where $I$ is the spatial inversion operator. We have checked numerically that the ground state is symmetric under $I$, while the first excited state is antisymmetric. We also see that they have very similar momentum densities and momentum-momentum correlations.

As we did for the ring, we can translate this into an approximate picture for the wave function of the ground and first excited states. Specifically, we propose

$$
\begin{aligned}
& \left|\Psi_{0}\right\rangle \simeq|C(\pi / 2)\rangle, \\
& \left|\Psi_{1}\right\rangle \simeq|S(\pi / 2)\rangle,
\end{aligned}
$$

with the coordinates in Eq. (26) referred to the midpoint between the walls. The adequacy of this approximation for some purposes can be inferred from an analysis of the approximate and numerically exact natural orbitals. It is possible to work out the reduced one-particle density matrix for $|C(k)\rangle$ and
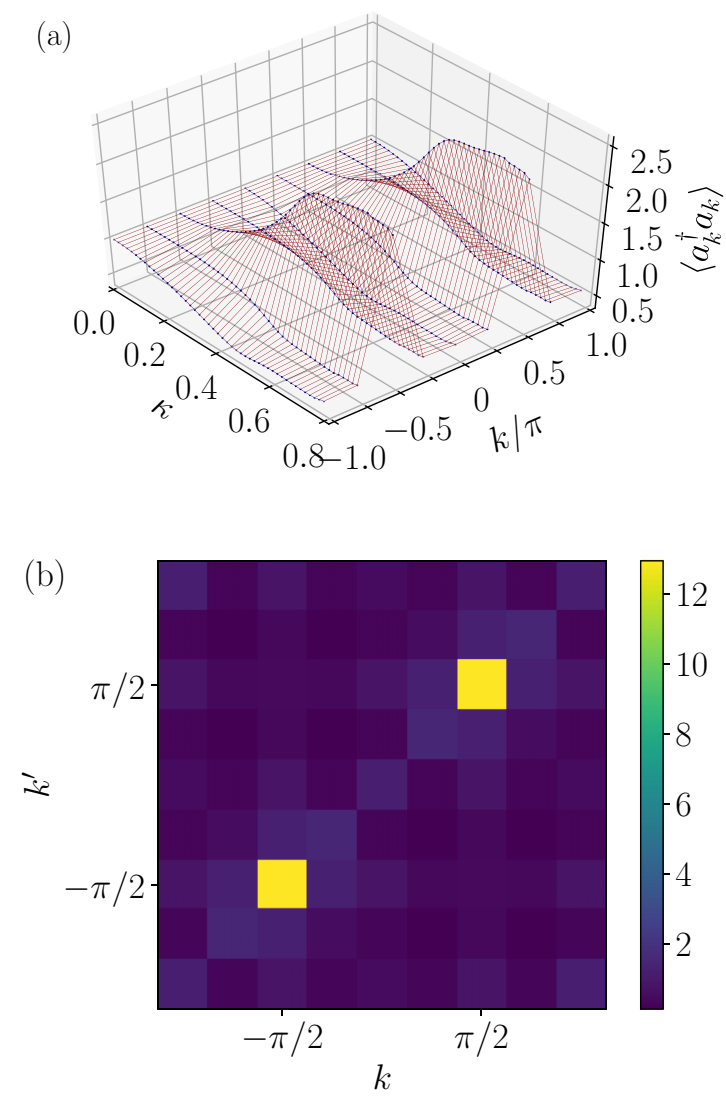

FIG. 9. (a) Momentum density for the ground state of $H_{\kappa}^{\mathrm{hw}}$ for eight particles on eight sites. Just as for the ring the momentum density develops distinct peaks at $k= \pm \pi / 2$. For $\kappa=0$, the system is in a Mott state. As $\kappa$ is increased two condensates with nonzero momenta form. (b) Momentum-momentum correlations at $\kappa=0.8$ for eight particles on eight sites. The isolated peaks at $\pm(\pi / 2, \pi / 2)$ indicate that the ground state is catlike.

$|S(k)\rangle$. One obtains

$$
\begin{aligned}
\rho^{(1)}\left(x, x^{\prime}\right) & =\frac{1}{L} \cos \left[k\left(x-x^{\prime}\right)\right] \\
& =\frac{1}{L}\left[\cos (k x) \cos \left(k x^{\prime}\right)+\sin (k x) \sin \left(k x^{\prime}\right)\right],
\end{aligned}
$$

from which we conclude that the natural orbitals are

$$
\phi_{0}(x)=\cos (k x), \quad \phi_{1}(x)=\sin (k x) .
$$

We can compare them with the natural orbitals obtained numerically. Setting $k=\pi / 2$, we find that they are indeed very similar. Figure 10 shows a direct comparison between the degenerate most occupied orbitals for eight particles on eight sites and the approximate natural orbitals in Eq. (45). We notice that the numerically exact state obeys the boundary conditions, so its orbitals go more gradually to zero close to the boundaries. One could ameliorate this by multiplying the truncated plane waves implicit in Eqs. (41) and (42) by an envelope function that smoothly goes to zero at the walls. This is also what in practice breaks the symmetry between 0 and $\pi$, since the envelope function's discontinuity at the walls will imbalance the momentum distribution. 


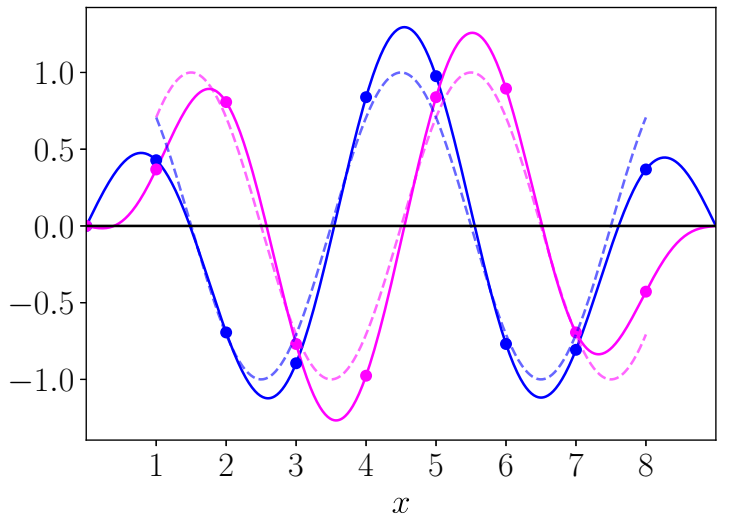

FIG. 10. The solid lines show the two most occupied natural orbitals at $\kappa=0.8$ for eight particles on eight sites. They are plotted continuously to better compare them with the analytically obtained orbitals from the ansatz in Eq. (41). The points indicate the value of the wave function at the real sites. They show very good agreement in the center of the lattice. At the edges, they start to disagree since the truncated waves are highly discontinuous at the edges while the stationary waves are not.

The foregoing analysis of symmetries and natural orbitals strongly supports the relevance of the approximate picture (41) proposed above. As we did for the ring, we can invert the terms and use the relations (25) to identify the cat branches between hard walls.

The fact that the ground state and first excited states are, respectively, even and odd under space inversion (always within the convention that, when expanded into momentum Fock states, the ground and first excited states have only real coefficients) strengthens the argument that our ground and first excited states are similar to (41) and (42).

If we understand that the ground state and the first excited state are, respectively, cosinelike and sinelike (with respect to the midpoint) we can expect the cat branches to be

$$
\left|\Psi_{ \pm}\right\rangle=\frac{1}{\sqrt{2}}\left(\left|\Psi_{0}\right\rangle \pm \mathrm{i}\left|\Psi_{1}\right\rangle\right) .
$$

This expectation is confirmed in Fig. 11, where the numerical momentum-momentum correlation is shown for the branches $\left|\Psi_{ \pm}\right\rangle$. As argued for the ring, the orthogonality of $\left|\Psi_{0}\right\rangle$ and $\left|\Psi_{1}\right\rangle$ guarantees $\left\langle\Psi_{+} \mid \Psi_{-}\right\rangle=0$.
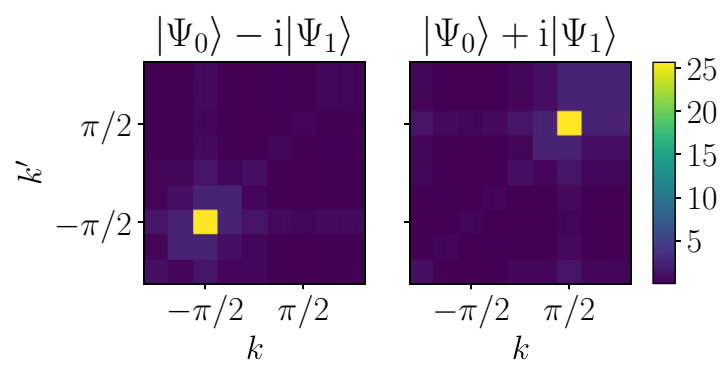

FIG. 11. Momentum correlations of the states $\left|\Psi_{ \pm}\right\rangle$constructed from the superposition of the ground and first excited states [see (46)] for $\kappa=0.8$. The isolated peaks clearly suggest that one can view the ground state as the superposition of two counter-propagating collective plane waves between the walls.

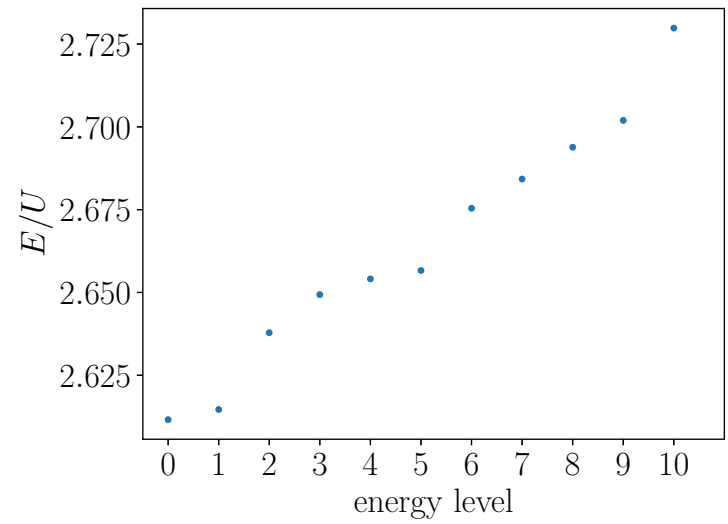

FIG. 12. Energy levels of ground and lowest-lying excited states for $\kappa=0.8$ with hard-wall confinement. The gap between the lowest two states and the rest of the spectrum is considerably smaller than for the ring case (Fig. 6).

The outcome is that for the hard-wall case the ground and first excited states can again be written as

$$
\begin{aligned}
& \left|\Psi_{0}\right\rangle=\frac{1}{\sqrt{2}}\left(\left|\Psi_{+}\right\rangle+\left|\Psi_{-}\right\rangle\right), \\
& \left|\Psi_{1}\right\rangle=\frac{-\mathrm{i}}{\sqrt{2}}\left(\left|\Psi_{+}\right\rangle-\left|\Psi_{-}\right\rangle\right),
\end{aligned}
$$

where $\left|\Psi_{ \pm}\right\rangle$represent complex but strictly orthonormal manybody configurations with a preferential occupation of momenta $\pm \pi / 2$. We identify them with the two orthonormal branches of the cat state. As for the ring case, we remark that the states $\left|\Psi_{ \pm}\right\rangle$yielding the simple-looking result of Fig. 11 actually involve thousands of momentum configurations.

Like for the ring case (see Sec. VIA), one might naively interpret the branches (46) as traveling many-body states. We also find here that the expectation value of the current operator [see Eq. (31)] vanishes in the ground doublet, with expressions identical to (33).

For completeness, we show in Fig. 12 the spectrum for the hard wall system. The lowest doublet is separated from the higher-lying excitations by a smaller energy interval than in the ring case. The comparison of the various energy differences in the ring and in the hard-wall cases takes us to the question of the fragility of the catlike correlations against finite-time state preparation, a problem which we address in Sec. IX.

\section{Harmonic confinement}

We have so far considered the case of a box potential, that is, a potential that is zero within two hard-wall boundaries. Although this type of trapping has been used in experiment [87], it is much more common to use a parabolic trap. To see how the presence of such a potential may modify the results we obtain, we add a potential term to the driven Bose-Hubbard model

$$
V=V_{0} \sum_{j}\left(x_{j}-x_{0}\right)^{2} n_{j},
$$

where $x_{0}$ is the center of the box and $n_{j}$ is the standard number operator. As before, we prepare the system in a perfect Mott 
state, and then add the above potential while slowly increasing the amplitude of the time-dependent driving.

For a weak trapping potential, $V_{0}=0.01 U$ and $J=U$, the momentum density function and the occupation of the natural orbitals strongly resemble the case of the flat trap $\left(V_{0}=0\right)$. As $\kappa$ is increased from zero, two natural orbitals become macroscopically occupied, while two peaks centered on momenta $\pm \pi / 2$ appear in the momentum density function. This provides strong evidence for the formation of a similar Schrödinger cat state. The appearance of the two peaks remains true for higher values of the trap curvature $\left(V_{0} / U=\right.$ 0.04 and 0.08 ) This is also true for random potentials; the cat features survive as long as the amplitude of the disorder potential is not large enough to localize the particles (not shown).

\section{MEASURES OF “CATTINESS"}

The question remains of how to quantify the quality of a catlike state, which may loosely be referred to as "cattiness" [88]. A number of cat quality measures have been proposed $[6,89,90]$. Quantitative measures tend to reward both the purity (branch orthogonality) and the size of the system. From the summary of Ref. [89] we may distinguish several approaches chosen to characterize the quality of an MQS state: definition of the effective size of the superposition by comparing it to a cat state made from two orthogonal modes [91-94], size of the relative particle-number fluctuations [78,95], entanglement measures taken from quantum information theory $[72,96,97]$, magnitude of the off-diagonal correlations to distinguish mixed states from cat states [7,8,74,98], and more measurement-based approaches [67,99-102].

In this section, in order to characterize the ground states found in our calculation, we adopt the pragmatic, measurement-based definition of cat quality introduced in [67]. Since we deal with pure many-body states involving many one-particle modes, this measure is particularly helpful to us because its general character is not restricted to the case of two modes. The measure in Ref. [67] is convenient for us also because we know the branches precisely. It attempts to quantify how well the two branches $A$ and $B$ of a superposition

$$
|\Psi\rangle=|A\rangle+|B\rangle
$$

can be distinguished after a measurement. More specifically it gauges the maximal probability of successfully inferring the two $n$-particle reduced density matrices ( $n$-PRDMs) $\rho_{A}^{(n)}$ and $\rho_{B}^{(n)}[103]$ related to the respective branches after an $n$-particle measurement:

$$
P^{(n)}=\frac{1}{2}+\frac{1}{4}\left\|\rho_{A}^{(n)}-\rho_{B}^{(n)}\right\|,
$$

where $\|\cdot\|$ is the trace norm $\|\rho\|=\sum_{i}\left|\lambda_{i}\right|$ with $\lambda_{i}$ the eigenvalues of $\rho$. If both density matrices are identical, then the probability of correctly inferring the branch from an $n$ particle measurement is $P=1 / 2$. This means one has not learned anything about either branch. For a perfect cat state $P^{(1)}=1$, which means an immediate "collapse" takes place after the first particle measurement. The more particles it takes to differentiate the branches, the smaller is the effective size of the superposition.
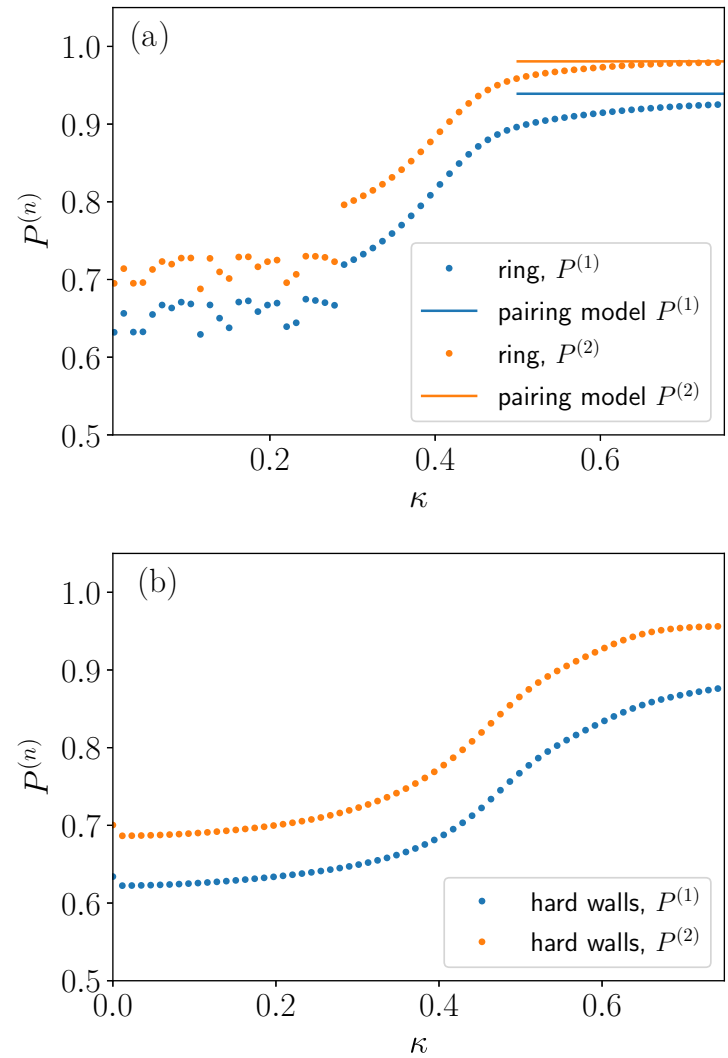

FIG. 13. Figure of merit based on the probability $P^{(n)}$ of knowing if the state collapsed into either branch after an $n$ particle measurement. (a) ring, (b) hard walls. The apparent discontinuity in the ring at $\kappa \simeq 0.3$ is due to a discontinuity in the behavior of the first excited state. The probabilities to infer the cat state for the hard-wall case are consistently lower than for the ring.

Here we simply evaluate (51) without linking it to an effective cat-size through a value of confidence as the authors of Ref. [67] did. We only consider up to two particle measurements. In the plane-wave representation, both branches are sharply defined by the splitting in Eq. (28).

The properly normalized components of the one-PRDM and two-PRDM are

$$
\begin{gathered}
\rho_{i l}^{(1)}=\frac{1}{N}\left\langle a_{k_{i}}^{\dagger} a_{k_{l}}\right\rangle, \\
\rho_{i j l m}^{(2)}=\frac{1}{N(N-1)}\left\langle a_{k_{i}}^{\dagger} a_{k_{j}}^{\dagger} a_{k_{l}} a_{k_{m}}\right\rangle .
\end{gathered}
$$

In Fig. 13, we present $P^{(n)}$ as a function of $\kappa$ for $n=1,2$. As $\kappa$ grows, it is evident that the probability of inferring $A$ or $B$ rises in both the ring and the hard walls. For $\kappa<0.75$, the biggest probabilities reached for the ring are $P^{(1)}=0.93$ and $P^{(2)}=$ 0.98 , whereas for the hard walls $P^{(1)}=0.88$ and $P^{(2)}=0.95$. In the ring one can see that the probabilities closely approach their theoretical limit, $P^{(1)}=0.94, P^{(2)}=0.98$, provided by the pairing model. Even in the large- $\kappa$ limit, the ground state is not a perfect cat, since the pairs in the reduction cloud are shared by both condensate branches. This effect seems to survive in the thermodynamic limit, as we argue below. 


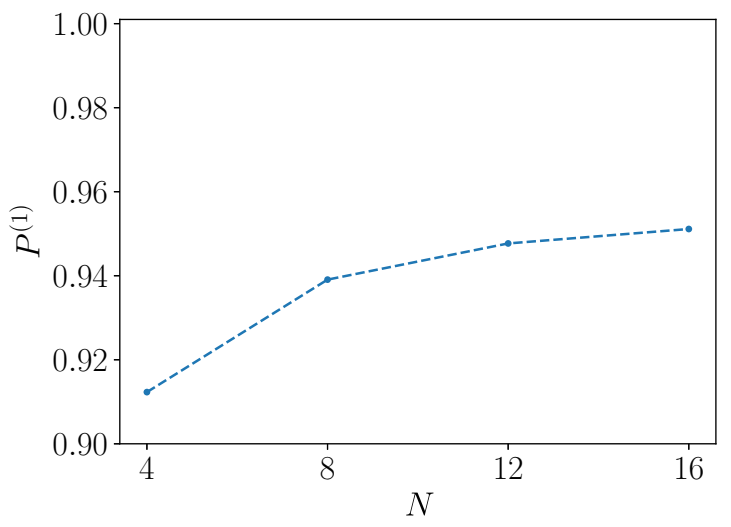

FIG. 14. Scaling of $P^{(1)}$ with system size for the pairing model. For up to 16 particles on 16 sites, the probability steadily increases.

As expected, the probability for collapsing the state after a two-particle measurement is consistently higher in both the ring and the hard-wall case. The apparent discontinuity in the ring for low $\kappa$ stems from a discontinuity of the first excited state, which does not develop its peaks at $\pm \pi / 2$ in the momentum density gradually (not shown).

Finally in Fig. 14, we look for the scaling of $P^{(1)}$ with growing system size. The pairing model predicts that $P^{(1)}$ increases with $N$ but saturates to a value below unity for $N \rightarrow \infty$ due to the presence of the reduction cloud. This result suggests that the weight of the reduction cloud saturates to a finite value in the thermodynamic limit.

Now we define a complementary figure of merit $C$ to quantify cattiness:

$$
C \equiv \sum_{k} p_{k}\left(\left|\left\langle A \mid \xi_{k}\right\rangle\right|-\left|\left\langle B \mid \xi_{k}\right\rangle\right|\right)^{2},
$$

where $|A\rangle$ and $|B\rangle$ are normalized, and

$$
p_{k}=\left\langle\Psi\left|n_{k}\right| \Psi\right\rangle / N
$$

is the probability of finding momentum $k$ in a one-particle measurement, and

$$
\left|\xi_{k}\right\rangle=\frac{n_{k}}{\mu_{k}^{2}}|\Psi\rangle
$$

with $\mu_{k}^{2} \equiv\left\langle\Psi\left|n_{k}^{2}\right| \Psi\right\rangle$, is the normalized many-boson state after projecting out configurations with momentum $k$ unoccupied. The cattiness $C$ is an intuitive measure tailored to our system. As in Ref. [67], we have looked for a figure of merit based on a measurement procedure. The idea is to infer the quality of the superposition by correlating it with the extent to which $\left|\xi_{k}\right\rangle$ overlaps with $|A\rangle$ or $|B\rangle$, i.e., with our ability to predict the outcome of the second one-particle measurement from the result of the first measurement.

For an ideal cat, $C=1$. For a noncat state $(|A\rangle=|B\rangle$ in Eq. (50)), $C=0$. In Fig. 15, we show the results for the ring and hard-wall boundary conditions. Again, the discontinuity for the ring stems from a discontinuity in the nature of the first excited state. In the Mott insulating regime, $C$ is very close to zero and increases notably after the transition. As for (51) we associate the fact that $C<1$ for large $\kappa$ to the presence of

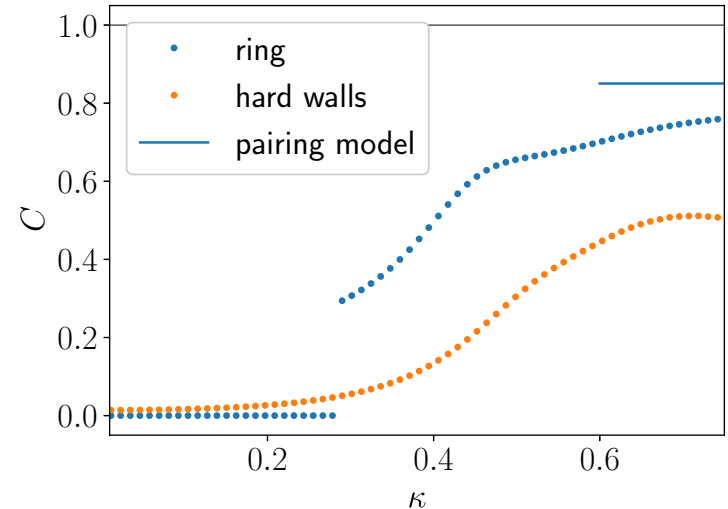

FIG. 15. Figure of merit for "cattiness" $C$ defined in Eq. (54). As before, the discontinuity in the ring result arises from the behavior of the first excited state. $C<1$ for large $\kappa$ because both branches share the reduction cloud. The largest values reached are: $C=0.79$ for the ring, $C=0.85$ for the pairing model, and $C=0.51$ for hard walls.

the reduction cloud, i.e., the multimode nature of the ground state.

The quantitative measure (54) is particularly simple to apply when only two modes are involved. As an illustration, in Appendix B, we explicitly calculate $C$ for a state of the form

$$
|\Psi\rangle=\frac{1}{K}(|A\rangle+|B\rangle)
$$

where $K$ normalizes $|\Psi\rangle$ and the branches are constructed from nonorthogonal single-particle orbitals:

$$
\begin{gathered}
|A\rangle=\frac{1}{\sqrt{N !}}\left(a^{\dagger}\right)^{N}|\mathrm{vac}\rangle, \\
|B\rangle=\frac{1}{\sqrt{N !}}\left[\cos (\theta) a^{\dagger}+\sin (\theta) b^{\dagger}\right]^{N}|\mathrm{vac}\rangle .
\end{gathered}
$$

For $\theta \in(0, \pi / 2)$, we find that $C$ nicely interpolates between 0 and 1.

\section{STATE PREPARATION}

We now consider how the cat state can actually be prepared in an experiment. As it is the ground state of the system, we might expect to naturally fall into this state as the system is cooled. However, the cat state consists of a superposition of two branches which are degenerate in energy and yield a splitting that vanishes in the thermodynamic limit. As a consequence, cooling the system towards zero temperature will just result in a classical mixture of the two branches [79]. We will instead look at the feasibility of preparing the cat state by adiabatic manipulation, as considered in Refs. [59,66]. Working with the full time-dependent Hamiltonian (1), we initialize the system in the Mott state, with one particle occupying each lattice site, and slowly ramp $\kappa$ up over many thousands of driving periods from an initial value of zero to a final value of $\kappa=0.8$, as shown in Fig. 16(a). When this final value is reached, we then hold $\kappa$ constant.

To give an indication of the degree to which the instantaneous state of the system exhibits cat correlations during this process, we evaluate the two-particle reduced density matrix at momenta $(\pi / 2, \pi / 2)$, that is, we compute $\chi \equiv\left\langle n_{\pi / 2} n_{\pi / 2}\right\rangle$. 


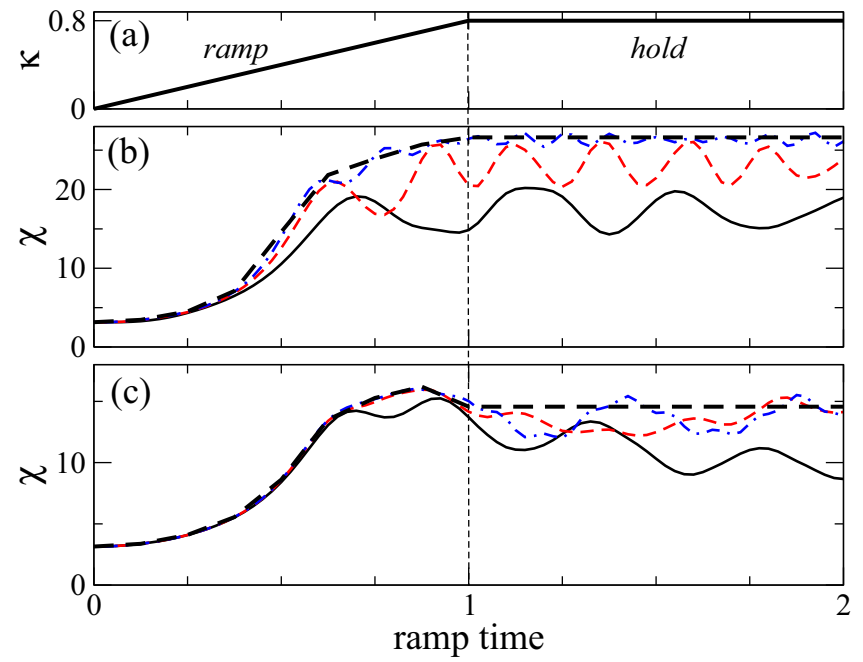

FIG. 16. The full time-dependent system is initialized in a Mott state, and $\kappa$ ramped linearly from 0 to a value of 0.8 over a timeinterval (the ramp time), and then held constant for the same time interval. (a) Time-dependence of the driving parameter, $\kappa$. (b) Cat correlation $\chi$ for a ring system. The solid (black) line is for a ramp time of $1600 T$, the thin dashed (red) line for $3200 T$ and the dot-dashed (blue) line for 6400T. As the ramp time is increased, the final value of $\chi$ becomes closer to that of the system's true ground state (thick dashed curve), and the oscillations reduce in amplitude, indicating that the cat state is being prepared with greater fidelity. (c) As in (b) but for a hard-wall system. The final value of $\chi$ is lower, and even for the slowest ramp time, the oscillations in $\chi$ remain significant. Much slower ramps would be needed to prepare the cat state with adequate fidelity. Physical parameters: $U=1, \omega=50$.

As we have seen earlier, this quantity has a large value when the system is in a catlike state and is small otherwise. So it acts as a reasonable alternative figure of merit to characterize the expected cat nature of the state we reach upon slowly ramping up the driving amplitude.

We show the behavior of $\chi$ for periodic boundary conditions (the ring) in Fig. 16(b), for various ramp speeds. It can clearly be seen that as the ramp speed decreases, the final value of $\chi$ increases, indicating that the cat state is being prepared with greater fidelity. This can be understood from the quasienergy spectrum of the system, shown in Fig. 17(a). For small $\kappa$, the system is a Mott insulator, and so the ground state is separated from the next excited states by a gap of order $U$. Accordingly, as long as the ramp speed is sufficiently slow with respect to the gap, $|\dot{\Delta}| / \Delta \ll 1$, the adiabatic approximation holds and the system safely remains in the ground state. Once the Mott gap closes, and the system becomes superfluid, the two lowest-lying states become an almost degenerate doublet. To form the cat correlations, we want the system to remain in this doublet, without being excited to higher excited states, which again imposes an adiabatic limit on the speed of the ramp (actually, as noted below, the system barely occupies the first excited state due to symmetry). As the gap is smaller here than in the Mott regime, the adiabaticity requirement is more stringent, and we can see it is only fulfilled when the ramp time is of the order of $6400 \mathrm{~T}$. For more rapid ramps, $\chi$ shows an oscillatory behavior arising
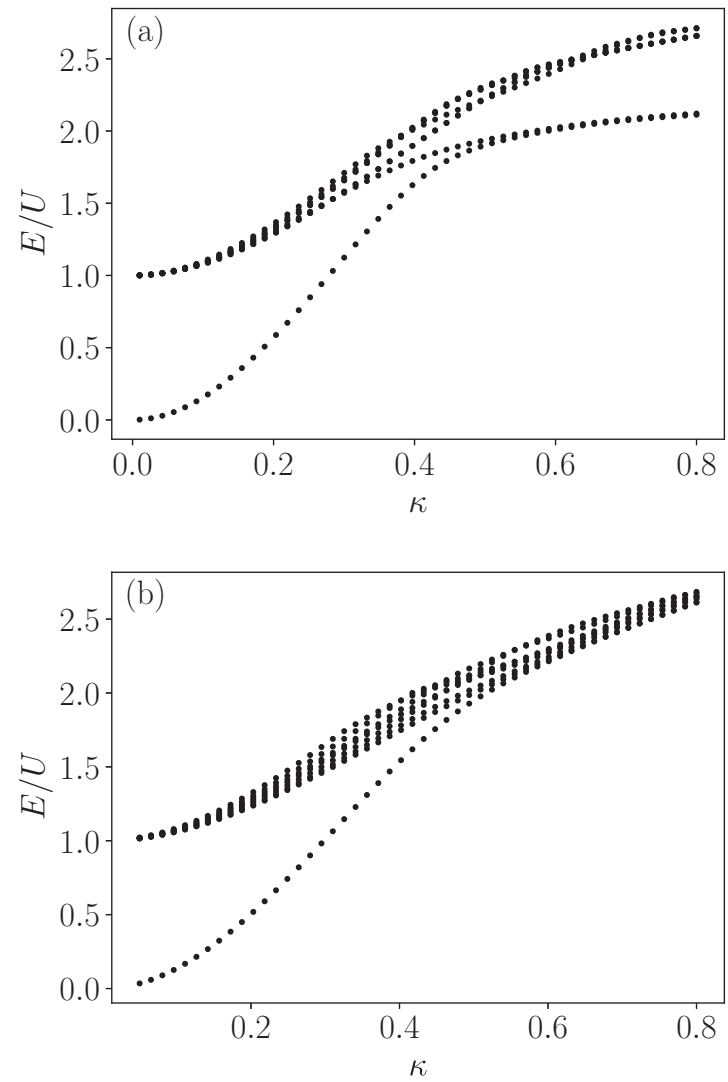

FIG. 17. Quasienergy spectrum as a function of $\kappa$ for (a) periodic boundary conditions, and (b) hard walls. Note how in (a) the groundstate manifold is always isolated from the rest of the spectrum, but that in (b) the ground-state merges into the spectrum as $\kappa$ increases. This makes adiabatic preparation of the cat state far more challenging for the case of hard walls.

from the excitation of higher states, which decreases the value of the cat correlations. To confirm this interpretation, we also measured the overlap (squared) of the final state with the true ground state of the system for $\kappa=0.8$. For a ramp time of $1600 T$, for example, this takes a value of 0.842 , indicating that a substantial proportion of the state has been excited out of the ground state. For the slowest ramp, however, this value rises to 0.999 , demonstrating that the procedure indeed has excellent fidelity.

In Fig. 16(c), we show the corresponding results for the hard-wall case. In contrast to the ring, however, even the slowest ramp speeds used are not able to prepare a cat state with comparable fidelity. As we show in Fig. 17(b), this is a consequence of the differences in the energy spectrum produced by the change in boundary conditions. For hard walls, the lowest doublet of states in the superfluid regime is barely separated from the next-highest states, as we can see explicitly by comparing Fig. 12 with Fig 6 . We can again measure the overlap of the obtained state with the actual ground state of the system, which reveals that even for a ramp time of $6400 T$ the fidelity is substantially lower $(\simeq 0.902)$ than for the corresponding case of the ring, As a result, slower ramp speeds would be needed to prepare a cat state, with time 
scales at least an order of magnitude longer than for the case of a ring.

Interestingly, we find that, for both the ring and the hardwall case, the overlap of the final state with any given excited state is very small. In particular, the overlap with the (also catlike) first excited state is essentially zero because the ramp up driving respects the symmetry of the ground state.

\section{RESILIENCE TO COLLAPSE}

Here we focus on the robustness of the cat state against collapse into one of its branches once it has been prepared. By collapse or decay of a cat state we understand its projection into one branch due to energy lowering or information retrieval and lasting for a very long time.

For a boson gas in a ring, one possible cause of collapse is the appearance of a spurious external flux. This can be due to a rotation drift of the optical lattice or to a departure from the ideal switching procedure [104] considered in Secs. III or IX. The spurious flux may be relevant in a SQUID [25,26], where the Josephson coupling generates a periodic flux dependence that can be tuned to form a double-well potential which in turn can be easily imbalanced. Here the physics is different. We have explicitly checked that, under the effect of an external flux, the two condensates shift their average momentum while preserving the cat structure. In the case of a box (hard wall), the situation is even simpler, as any velocity drift can be gauged away.

Particle losses can be a cause of MQS collapse [59,81]. A necessary condition for this to be an efficient decay mechanism is that the emitted atom carries information on the cat branch it comes from. Assuming that upon detection it is possible to know the momentum the atom had in the optical lattice, the information retrieval might be sufficient to cause the collapse. However, this is not clearly the case if we note that the atom with nonzero momentum in the lattice had actually zero group velocity before being emitted (see Sec. VIA). For the reduction cloud, the situation is more radical: even if we were able to know the initial crystal momentum of the detected atom, if this momentum happens to belong to the reduction cloud (i.e., it is different from $\pm \pi / 2$ ), then it carries no information whatsoever on the branch state, since the reduction cloud is identically shared by the two $\pm \pi / 2$-momentum condensates.

A similar discussion of atom losses applies to a kinetically driven boson gas between hard walls.

The interaction with the thermal cloud has sometimes been identified as a possible cause of cat state collapse [66]. Here we have not performed a study of thermal excitations, but the existing experience on conventional boson gases teaches that quasiparticles often reflect the structure of the depletion cloud. The fact that the reduction cloud is common to the two macroscopic condensates strongly suggests that the same is true for the quasiparticles. Thus the assertion that the thermal cloud cannot cause the decay of the MQS state may be viewed as an educated conjecture.

Diagonal impurities may also break the nice degeneracy between cat branches. Both for impurity disorder (see Sec. IX) and for isolated impurities, we have numerically checked that the cat structure remains intact within a range of nonzero impurity strength (not shown).

Finally, one may reflect on the role of standard decoherence, i.e., dephasing caused by coupling to an external dissipative environment [2-5]. Cold atom systems tend to be isolated and thus insensitive to external sources of dissipation. For the present system, there is an additional, more profound reason to believe that dynamic decoherence is not operating here. A necessary condition for an environment to cause the collapse of a cat state is that it couples to the system observable whose eigenstates (the "pointer basis" of Ref. [3]) characterize the cat branches. It is difficult to think of a dissipative environment that meets those requirements, considering that the branches of the present state collectively populate nonzero momentum states while yielding individually a zero current average, due to the counterintuitive current operator (see Sec. VI A).

Altogether, there seems to be a number of reasons for asserting that the cat states we have identified in the ground doublet of a kinetically driven boson gas are more protected than most cat states so far investigated. Although we have not been able to check it explicitly, it seems reasonable to venture that this unusual MQS robustness will survive in the thermodynamic limit. Thus the picture emerges of a many-body state with a practically hidden cat structure that only blossoms when subject to the invasive momentum-measurement of a time-of-flight experiment.

\section{CONCLUSIONS}

In this paper we have investigated a novel type of Schrödinger cat state whose main characteristic is its unusual resilience to collapse into one of its branches. This is the case of a one-dimensional boson gas (described by the $\mathrm{BH}$ model) between hard walls subject to time-periodic driving of the kinetic energy with zero average. As noted in Ref. [16], such a boson system system has a preference for collectively populating the states of momentum $\pm \pi / 2$.

We have focused on exploring the catlike nature of the ground state, which has led us to a deeper understanding of this atypical many-body problem. This has been possible thanks to the fortunate fact that, in the ring case and for medium and large driving amplitudes, the time-independent effective Hamiltonian can be approximated by a Hamiltonian which resembles the Richardson-Gaudin pairing model. Our effective system additionally includes an attractive interaction in momentum-space [19] and a peculiar pairing rule that links momenta $k, \pi-k$ (instead of the conventional $k,-k$ ). This unusual pairing interaction gives rise to a modified depletion cloud which is shared by the two current-carrying condensate branches and which we label reduction cloud to clearly distinguish it from the qualitatively different depletion cloud of the conventional, undriven BH model.

The simplified toy model just described does not apply so well to the boson gas between hard walls. Yet many of its features can still be qualitatively understood in terms of an ensemble of atoms with highly correlated momenta. This picture is quantitatively confirmed by analyzing the manybody ground state in the representation of truncated plane waves (see Fig. 11). We have also studied the case of harmonic 
confinement and found the same structure of many atoms with a high momentum correlation, which underlines the robustness of the effect.

We have investigated aspects of the ground state such as its intrinsic quality as a cat state, the feasibility of its preparation, and its resistance to collapse. The emerging picture is that of a boson system whose ground doublet is formed by two catlike states. From the ground state we can assert that its cat quality and preparation feasibility are acceptable and its resilience to collapse remarkable.

The cat branches differ in the momentum that is macroscopically occupied. Despite their nonzero momentum, each cat branch has separately a zero average current. This counterintuitive result is directly linked to the exotic character of the effective Hamiltonian, which results in an also atypical current operator. The atom momenta can be measured in a time-offlight experiment, since upon removal of the confining optical lattice the crystal momentum within the lattice becomes the linear momentum in free space. In such an experiment, the majority of atoms are expected to flock in a given direction that varies randomly from run to run. In such a scenario, one could investigate the statistics of correlations between detected momenta at different times, thus probing also the reduction cloud of the fragmented condensate and its resulting correlations.

In the presence of a finite effective flux, the kinetically driven boson gas displays a nonzero current whose sheer existence depends crucially on the presence of the reduction cloud. This may be viewed as a new form of superfluidity.

The present work paves the way to the study of a novel class of low-energy robust cat states that can be realized in cold atom setups and other similar systems.

\section{ACKNOWLEDGMENTS}

We would like to thank Anthony Leggett and Peter Zoller for valuable discussions. This work has been supported by Spain's MINECO through Grants No. FIS2013-41716-P and FIS2017-84368-P. One of us (FS) would like to acknowledge the support of the Real Colegio Complutense at Harvard and the Harvard-MIT Center for Ultracold Atoms, where part of this work was done.

\section{APPENDIX A: VARIATIONAL CALCULATION}

Take the state

$$
\begin{aligned}
|\Psi(\alpha)\rangle= & \alpha|F\rangle+\beta|R\rangle \\
= & \frac{\alpha}{\sqrt{2}}\left(\left|N_{-\frac{\pi}{2}}, 0\right\rangle+\left|0, N_{\frac{\pi}{2}}\right\rangle\right) \\
& +\frac{\beta}{\sqrt{2(L-2)}}\left(\left|(N-2)_{-\frac{\pi}{2}}, 0_{\frac{\pi}{2}}\right\rangle\right. \\
& \left.+\left|0_{-\frac{\pi}{2}},(N-2)_{\frac{\pi}{2}}\right\rangle\right) \sum_{k \neq \pm \frac{\pi}{2}}\left|1_{k}, 1_{\pi-k}\right\rangle,
\end{aligned}
$$

where $\alpha, \beta$ are real and $\alpha^{2}+\beta^{2}=1$. Here $F$ stands for fragmented condensates and $R$ for reduction cloud. We find

$$
\begin{aligned}
E(\alpha) & =\left\langle\Psi(\alpha)\left|h_{\infty}\right| \Psi(\alpha)\right\rangle \\
& =\alpha^{2}\left(E_{F}-E_{R}\right)+\alpha \sqrt{1-\alpha^{2}} V+E_{R},
\end{aligned}
$$

which is minimized for $\alpha=\alpha_{0}$ with

$$
\alpha_{0}=-\operatorname{sgn}(V) \sqrt{\frac{1}{2}+\left(1+\frac{2 V^{2}}{\left(E_{F}-E_{R}\right)^{2}}\right)^{-1 / 2}} .
$$

The various constants are

$$
\begin{aligned}
E_{F} & =\left\langle F\left|h_{\infty}\right| F\right\rangle=-N^{2}, \\
V & =2\left\langle F\left|h_{\infty}\right| R\right\rangle \\
& =\sqrt{N(N-1)(L-2)}, \\
E_{R} & =\left\langle R\left|h_{\infty}\right| R\right\rangle \\
& =-N^{2}+4 N+L-9 .
\end{aligned}
$$

For unit filling $(N=L)$ the energy reduction for eight particles on eight sites due to the cloud is $E(\alpha)-E_{R}=-8.19$ (here, $E_{F}=-64, E\left(\alpha_{0}\right)=-72.2$ and the numerically exact ground state energy is $\left.E_{\mathrm{gs}}=-83.2\right)$. For $N>9$, one can prove generally that

$$
E(\alpha)<E_{F} .
$$

In the limit of large $N$, one obtains

$$
E\left(\alpha_{0}\right)-E_{F}=-N \sqrt{N} .
$$

This clearly shows how the mixing with a cloud of pairs lowers the energy of the fully occupied modes $\pm \pi / 2$. For simplicity we have omitted the inclusion of $\mid(N-$ $\left.2)_{-\pi / 2}, 2_{\pi / 2}\right\rangle,\left|2_{-\pi / 2},(N-2)_{\pi / 2}\right\rangle$ because it would have caused the introduction of yet another independent parameter, without further elucidating the effect of interactions of the condensate with its cloud.

In order to better understand the benefit of macroscopically occupying a single mode, we may choose a different state as a starting point for the variational calculation. Specifically, we take the "center" configuration in the expansion of Eq. (8) and show how it benefits less from the reduction cloud:

$$
\begin{aligned}
\left|\Psi_{\mathrm{c}}(\alpha)\right\rangle= & \alpha|\tilde{F}\rangle+\beta|\tilde{R}\rangle \\
= & \alpha\left|(N / 2)_{-\frac{\pi}{2}},(N / 2)_{\frac{\pi}{2}}\right\rangle \\
& +\frac{\beta}{\sqrt{2(L-2)}}\left(\left|(N / 2-2)_{-\frac{\pi}{2}},(N / 2)_{\frac{\pi}{2}}\right\rangle\right. \\
& \left.+\left|(N / 2)_{-\frac{\pi}{2}},(N / 2-2)_{\frac{\pi}{2}}\right|\right) \sum_{k \neq \pm \frac{\pi}{2}}\left|1_{k}, 1_{\pi-k}\right\rangle .
\end{aligned}
$$

When comparing (A12) with (A2), the key difference is that the two condensate terms in the reduction cloud of (A12) can mix due to the pair interaction term, namely,

$$
\begin{aligned}
& \left\langle(N / 2-2)_{-\frac{\pi}{2}},(N / 2)_{\frac{\pi}{2}}\left|h_{\infty}\right|(N / 2)_{-\frac{\pi}{2}},(N / 2-2)_{\frac{\pi}{2}}\right\rangle \\
& \quad=N / 2(N / 2-1) \neq 0 .
\end{aligned}
$$

By contrast, this mixing does not affect (A2) because their counterparts are not connected by $h_{\infty}$ :

$$
\left\langle(N-2)_{-\frac{\pi}{2}}, 0_{\frac{\pi}{2}}\left|h_{\infty}\right| 0_{-\frac{\pi}{2}},(N-2)_{\frac{\pi}{2}}\right\rangle=0 .
$$


Again we minimize

$$
\tilde{E}(\alpha)=\alpha^{2}\left(E_{\tilde{F}}-E_{\tilde{R}}\right)+\alpha \sqrt{1-\alpha^{2}} \tilde{V}+E_{\tilde{R}},
$$

where

$$
\begin{aligned}
E_{\tilde{F}} & =\left\langle\tilde{F}\left|h_{\infty}\right| \tilde{F}\right\rangle=-N^{2} / 2, \\
\tilde{V} & =2\left\langle\tilde{F}\left|h_{\infty}\right| \tilde{R}\right\rangle \\
& =\sqrt{N(N / 2-1)(L-2),} \\
E_{\tilde{R}} & =\left\langle\tilde{R}\left|h_{\infty}\right| \tilde{R}\right\rangle \\
& =-N^{2} / 4+3 N / 2+L-9 .
\end{aligned}
$$

The value $\tilde{\alpha}_{0}$ that minimizes $\tilde{E}(\alpha)$ is formally the same as (A5) but with Eqs. (A6)-(A8) replaced by (A16) and (A17).

Not only is $\tilde{E}\left(\tilde{\alpha}_{0}\right)>E\left(\alpha_{0}\right)$ but also the contribution of the reduction cloud to the state and the energy is altered. For unit filling $N=L$, the change introduced to $E_{\tilde{R}}$ due to the mixing in Eq. (A13) causes $\lim _{L \rightarrow \infty} \tilde{\alpha}_{0}^{2}=1$ as opposed to $\lim _{L \rightarrow \infty} \alpha_{0}^{2}=1 / 2$. The closer $\tilde{\alpha}_{0}$ is to one, the less important is the contribution of the reduction cloud to $\left|\Psi_{c}\right\rangle$ and to $\tilde{E}_{\tilde{\alpha}_{0}}$, as can be seen in Eq. (A15) and the normalization condition for $\alpha, \beta$. This means that the reduction cloud in $\left|\Psi_{c}\left(\tilde{\alpha}_{0}\right)\right\rangle$ loses importance once the system size increases, which is not the case for $\left|\Psi\left(\alpha_{0}\right)\right\rangle$. Even for moderate sizes, such as eight particles on eight sites $\tilde{\alpha}_{0}^{2}=0.92$, whereas $\alpha_{0}^{2}=0.76$ (the bigger $\alpha$ the smaller the weight of the reduction cloud). We note that (A2) and (A12) are just approximate ansätze, hence the weight of the condensate does not need to vanish in the thermodynamic limit, as expected for an exact description.

\section{APPENDIX B: CAT FIGURE OF MERIT}

We calculate $C$ for one of the states proposed in Ref. [91]

$$
|\Psi\rangle=\frac{1}{K}(|A\rangle+|B\rangle),
$$

where

$$
\begin{gathered}
|A\rangle=|N, 0\rangle \\
|B\rangle=\frac{1}{\sqrt{N !}}\left(c_{\theta} a^{\dagger}+s_{\theta} b^{\dagger}\right)^{N}|\mathrm{vac}\rangle \\
=\frac{1}{\sqrt{N !}} \sum_{l}\left(\begin{array}{c}
N \\
l
\end{array}\right) c_{\theta}^{l} s_{\theta}^{N-l} \sqrt{l !(N-l) !|l, N-l\rangle,}
\end{gathered}
$$

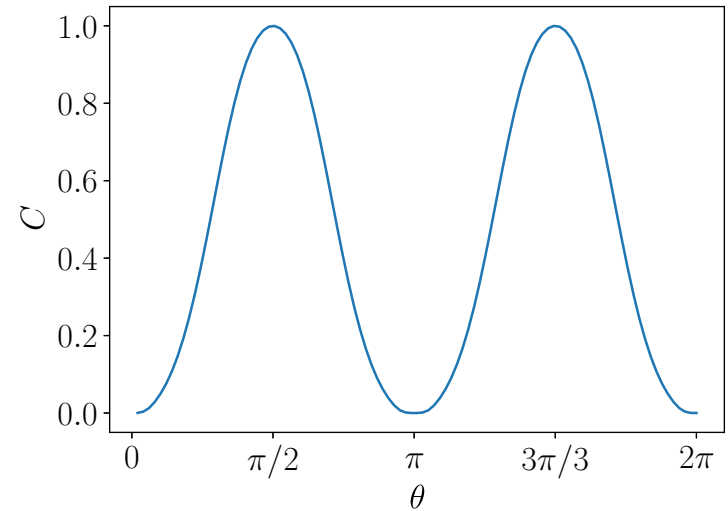

FIG. 18. Cattiness $C$ for (B1). For $\theta=\pi / 2,3 \pi / 2$ the state is a perfect cat, for $\theta=0, \pi$ it has no cat state feature.

where $c_{\theta} \equiv \cos \theta$ and $s_{\theta} \equiv \sin \theta$. We find

$$
\begin{aligned}
C(\theta) & =\sum_{k} f_{k}\left(\left|\left\langle A \mid \xi_{k}\right\rangle\right|-\left|\left\langle B \mid \xi_{k}\right\rangle\right|\right)^{2} \\
& =\frac{1}{N K^{4}}\left(\frac{1}{\mathcal{M}_{1}^{2}}\left(N+2 N c_{\theta}^{N}+g_{1}\right)\left(N-g_{1}\right)^{2}+\frac{g_{2}^{3}}{\mathcal{M}_{2}^{2}}\right),
\end{aligned}
$$

where

$$
\begin{aligned}
\mathcal{M}_{1}^{2}= & \frac{1}{K^{2}}\left(N^{2}+2 N^{2} c_{\theta}^{N}\right. \\
& \left.+\frac{1}{N !} \sum_{l=0}^{N}\left(\begin{array}{c}
N \\
l
\end{array}\right)^{2} c_{\theta}^{2 l} s_{\theta}^{2(N-l)} l^{2} l !(N-l) !\right), \\
\mathcal{M}_{2}^{2}= & \frac{1}{N ! K^{2}} \sum_{l=0}^{N}\left(\begin{array}{c}
N \\
l
\end{array}\right)^{2} c_{\theta}^{2 l} s_{\theta}^{2(N-l)}(N-l)^{2} l !(N-l) !
\end{aligned}
$$

and

$$
\begin{gathered}
g_{1}=\frac{1}{N !} \sum_{l=0}^{N}\left(\begin{array}{c}
N \\
l
\end{array}\right)^{2} c_{\theta}^{2 l} s_{\theta}^{2(N-l)} l !(N-l) ! l, \\
g_{2}=\frac{1}{N !} \sum_{l=0}^{N}\left(\begin{array}{c}
N \\
l
\end{array}\right)^{2} c_{\theta}^{2 l} s_{\theta}^{2(N-l)} l !(N-l) !(N-l) .
\end{gathered}
$$

We present a plot of $C$ in Fig. 18.
[1] E. Schrödinger, Die gegenwärtige Situation in der Quantenmechanik, Naturwissenschaften 23, 823 (1935).

[2] J. Von Neumann, Mathematische Grundlagen der Quantenmechanik, Grundlehren der mathematischen Wissenschaften (Springer, Berlin, 1932).

[3] W. H. Zurek, Environment-induced superselection rules, Phys. Rev. D 26, 1862 (1982).

[4] E. Joos and H. D. Zeh, The emergence of classical properties through interaction with the environment, Z. Phys. B 59, 223 (1985).
[5] W. H. Zurek, Decoherence and the transition from quantum to classical, Phys. Today 44, 36 (1991).

[6] A. J. Leggett, Testing the limits of quantum mechanics: motivation, state of play, prospects, J. Phys.: Condens. Matter 14, R415 (2002).

[7] E. G. Cavalcanti and M. D. Reid, Criteria for generalized macroscopic and mesoscopic quantum coherence, Phys. Rev. A 77, 062108 (2008).

[8] B. Opanchuk, L. Rosales-Zárate, R. Y. Teh, and M. D. Reid, Quantifying the mesoscopic quantum coherence of 
approximate NOON states and spin-squeezed two-mode BoseEinstein condensates, Phys. Rev. A 94, 062125 (2016).

[9] G. C. Knee, K. Kakuyanagi, M.-C. Yeh, Y. Matsuzaki, H. Toida, H. Yamaguchi, S. Saito, A. J. Leggett, and W. J. Munro, A strict experimental test of macroscopic realism in a superconducting flux qubit, Nat. Commun. 7, 13253 (2016).

[10] M. D. Reid, Bell inequalities for falsifying mesoscopic local realism via amplification of quantum noise, Phys. Rev. A 97, 042113 (2018).

[11] J. Higbie and D. M. Stamper-Kurn, Generating macroscopicquantum-superposition states in momentum and internal-state space from Bose-Einstein condensates with repulsive interactions, Phys. Rev. A 69, 053605 (2004).

[12] J. J. Bollinger, W. M. Itano, D. J. Wineland, and D. J. Heinzen, Optimal frequency measurements with maximally correlated states, Phys. Rev. A 54, R4649 (1996).

[13] D. Leibfried, E. Knill, S. Seidelin, J. Britton, R. B. Blakestad, J. Chiaverini, D. B. Hume, W. M. Itano, J. D. Jost, C. Langer, R. Ozeri, R. Reichle, and D. J. Wineland, Creation of a six-atom 'Schrödinger cat' state, Nature (London) 438, 639 (2005).

[14] V. Giovannetti, S. Lloyd, and L. Maccone, Quantum-enhanced measurements: beating the standard quantum limit, Science 306, 1330 (2004).

[15] J. A. Wheeler and W. H. Zurek, Quantum Theory and Measurement (Princeton University Press, Princeton, NJ, 2014).

[16] G. Pieplow, F. Sols, and C. Creffield, Generation of atypical hopping and interactions by kinetic driving, New J. Phys. 20, 073045 (2018).

[17] R. W. Richardson, Exact eigenstates of the pairing-force Hamiltonian. II, J. Math. Phys. 6, 1034 (1965).

[18] J. Dukelsky, S. Pittel, and G. Sierra, Colloquium: Exactly solvable Richardson-Gaudin models for many-body quantum systems, Rev. Mod. Phys. 76, 643 (2004).

[19] M. Heimsoth, C. E. Creffield, L. D. Carr, and F. Sols, Orbital Josephson effect and interactions in driven atom condensates on a ring, New J. Phys. 14, 075023 (2012).

[20] F. Sols, Randomization of the phase after suppression of the Josephson coupling, Phys. B: Condens. Matter 194-196, 1389 (1994).

[21] M. Greiner, O. Mandel, T. W. Hänsch, and I. Bloch, Collapse and revival of the matter wave field of a Bose-Einstein condensate, Nature (London) 419, 51 (2002).

[22] I. Zapata, F. Sols, and A. J. Leggett, Phase dynamics after connection of two separate Bose-Einstein condensates, Phys. Rev. A 67, 021603(R) (2003).

[23] C. Monroe, D. M. Meekhof, B. E. King, and D. J. Wineland, A Schrödinger cat superposition state of an atom, Science 272, 1131 (1996).

[24] M. Brune, E. Hagley, J. Dreyer, X. Maître, A. Maali, C. Wunderlich, J. M. Raimond, and S. Haroche, Observing the Progressive Decoherence of the "Meter" in a Quantum Measurement, Phys. Rev. Lett. 77, 4887 (1996).

[25] C. H. van der Wal, A. C. J. ter Haar, F. K. Wilhelm, R. N. Schouten, C. J. P. M. Harmans, T. P. Orlando, S. Lloyd, and J. E. Mooij, Quantum superposition of macroscopic persistentcurrent states, Science 290, 773 (2000).

[26] J. R. Friedman, V. Patel, W. Chen, S. K. Tolpygo, and J. E. Lukens, Quantum superposition of distinct macroscopic states, Nature (London) 406, 43 (2000).
[27] D. Esteve, J. M. Martinis, C. Urbina, E. Turlot, M. H. Devoret, H. Grabert, and S. Linkwitz, Observation of the temporal decoupling effect on the macroscopic quantum tunneling of a Josephson junction, Phys. Scr. 1989, 121 (1989).

[28] P. Silvestrini, V. G. Palmieri, B. Ruggiero, and M. Russo, Observation of Energy Levels Quantization in Underdamped Josephson Junctions Above the Classical-Quantum Regime Crossover Temperature, Phys. Rev. Lett. 79, 3046 (1997).

[29] R. Rouse, S. Han, and J. E. Lukens, Observation of Resonant Tunneling between Macroscopically Distinct Quantum Levels, Phys. Rev. Lett. 75, 1614 (1995).

[30] Y. Nakamura, Y. A. Pashkin, and J. S. Tsai, Coherent control of macroscopic quantum states in a single-Cooper-pair box, Nature (London) 398, 786 (1999).

[31] M. Arndt, O. Nairz, J. Vos-Andreae, C. Keller, G. Van der Zouw, and A. Zeilinger, Wave-particle duality of $\mathrm{C}_{60}$ molecules, Nature (London) 401, 680 (1999).

[32] O. Nairz, M. Arndt, and A. Zeilinger, Quantum interference experiments with large molecules, Am. J. Phys. 71, 319 (2003).

[33] S. Eibenberger, S. Gerlich, M. Arndt, M. Mayor, and J. Tüxen, Matter-wave interference of particles selected from a molecular library with masses exceeding 10000 amu, Phys. Chem. Chem. Phys. 15, 14696 (2013).

[34] S. Gerlich, S. Eibenberger, M. Tomandl, S. Nimmrichter, K. Hornberger, P. J. Fagan, J. Tüxen, M. Mayor, and M. Arndt, Quantum interference of large organic molecules, Nat. Commun. 2, 263 (2011).

[35] D. D. Awschalom, J. F. Smyth, G. Grinstein, D. P. DiVincenzo, and D. Loss, Macroscopic Quantum Tunneling in Magnetic Proteins, Phys. Rev. Lett. 68, 3092 (1992).

[36] E. M. Chudnovsky, Macroscopic quantum tunneling of the magnetic moment, J. Appl. Phys. 73, 6697 (1993).

[37] J. R. Friedman, M. P. Sarachik, J. Tejada, and R. Ziolo, Macroscopic Measurement of Resonant Magnetization Tunneling in High-Spin Molecules, Phys. Rev. Lett. 76, 3830 (1996).

[38] C. A. Sackett, D. Kielpinski, B. E. King, C. Langer, V. Meyer, C. J. Myatt, M. Rowe, Q. A. Turchette, W. M. Itano, D. J. Wineland, and C. Monroe, Experimental entanglement of four particles, Nature (London) 404, 256 (2000).

[39] T. Monz, P. Schindler, J. T. Barreiro, M. Chwalla, D. Nigg, W. A. Coish, M. Harlander, W. Hänsel, M. Hennrich, and R. Blatt, 14-Qubit Entanglement: Creation and Coherence, Phys. Rev. Lett. 106, 130506 (2011).

[40] A. Ourjoumtsev, R. Tualle-Brouri, J. Laurat, and P. Grangier, Generating optical Schrödinger kittens for quantum information processing, Science 312, 83 (2006).

[41] A. Ourjoumtsev, H. Jeong, R. Tualle-Brouri, and P. Grangier, Generation of optical Schrödinger cats from photon number states, Nature (London) 448, 784 (2007).

[42] G. Kirchmair, B. Vlastakis, Z. Leghtas, S. E. Nigg, H. Paik, E. Ginossar, M. Mirrahimi, L. Frunzio, S. M. Girvin, and R. J. Schoelkopf, Observation of quantum state collapse and revival due to the single-photon Kerr effect, Nature (London) 495, 205 (2013)

[43] C. Wang, Y. Y. Gao, P. Reinhold, R. W. Heeres, N. Ofek, K. Chou, C. Axline, M. Reagor, J. Blumoff, K. M. Sliwa, L. Frunzio, S. M. Girvin, L. Jiang, M. Mirrahimi, M. H. Devoret, and R. J. Schoelkopf, A Schrödinger cat living in two boxes, Science 352, 1087 (2016). 
[44] N. Bruno, A. Martin, P. Sekatski, N. Sangouard, R. T. Thew, and N. Gisin, Displacement of entanglement back and forth between the micro and macro domains, Nat. Phys. 9, 545 (2013).

[45] A. I. Lvovsky, R. Ghobadi, A. Chandra, A. S. Prasad, and C. Simon, Observation of micro-macro entanglement of light, Nat. Phys. 9, 541 (2013).

[46] B. Vlastakis, G. Kirchmair, Z. Leghtas, S. E. Nigg, L. Frunzio, S. M. Girvin, M. Mirrahimi, M. H. Devoret, and R. J. Schoelkopf, Deterministically encoding quantum information using 100-photon Schrödinger cat states, Science 342, 607 (2013).

[47] A. D. O'Connell, M. Hofheinz, M. Ansmann, R. C. Bialczak, M. Lenander, E. Lucero, M. Neeley, D. Sank, H. Wang, M. Weides, J. Wenner, J. M. Martinis, and A. N. Cleland, Quantum ground state and single-phonon control of a mechanical resonator, Nature (London) 464, 697 (2010).

[48] N. Kiesel, F. Blaser, U. Delić, D. Grass, R. Kaltenbaek, and M. Aspelmeyer, Cavity cooling of an optically levitated submicron particle, Proc. Natl. Acad. Sci. USA 110, 14180 (2013).

[49] D. L. Haycock, P. M. Alsing, I. H. Deutsch, J. Grondalski, and P. S. Jessen, Mesoscopic Quantum Coherence in an Optical Lattice, Phys. Rev. Lett. 85, 3365 (2000).

[50] A. J. Leggett and A. Garg, Quantum Mechanics Versus Macroscopic Realism: Is the Flux there when Nobody Looks? Phys. Rev. Lett. 54, 857 (1985).

[51] A. D. Armour, M. P. Blencowe, and K. C. Schwab, Entanglement and Decoherence of a Micromechanical Resonator Via Coupling to a Cooper-Pair Box, Phys. Rev. Lett. 88, 148301 (2002).

[52] S. Bose, K. Jacobs, and P. L. Knight, Scheme to probe the decoherence of a macroscopic object, Phys. Rev. A 59, 3204 (1999).

[53] W. Marshall, C. Simon, R. Penrose, and D. Bouwmeester, Towards Quantum Superpositions of a Mirror, Phys. Rev. Lett. 91, 130401 (2003).

[54] Y. Shen, S. M. Assad, N. B. Grosse, X. Y. Li, M. D. Reid, and P. K. Lam, Nonlinear Entanglement and its Application to Generating Cat States, Phys. Rev. Lett. 114, 100403 (2015).

[55] J.-Q. Liao, J.-F. Huang, and L. Tian, Generation of macroscopic Schrödinger-cat states in qubit-oscillator systems, Phys. Rev. A 93, 033853 (2016).

[56] S. Bose, K. Jacobs, and P. L. Knight, Preparation of nonclassical states in cavities with a moving mirror, Phys. Rev. A 56, 4175 (1997).

[57] B. Yurke and D. Stoler, Generating Quantum Mechanical Superpositions of Macroscopically Distinguishable States Via Amplitude Dispersion, Phys. Rev. Lett. 57, 13 (1986).

[58] S.-B. Zheng, One-Step Synthesis of Multiatom GreenbergerHorne-Zeilinger States, Phys. Rev. Lett. 87, 230404 (2001).

[59] J. I. Cirac, M. Lewenstein, K. Mølmer, and P. Zoller, Quantum superposition states of Bose-Einstein condensates, Phys. Rev. A 57, 1208 (1998).

[60] J. Ruostekoski, M. J. Collett, R. Graham, and D. F. Walls, Macroscopic superpositions of Bose-Einstein condensates, Phys. Rev. A 57, 511 (1998).

[61] D. A. R. Dalvit, J. Dziarmaga, and W. H. Zurek, Decoherence in Bose-Einstein condensates: Towards bigger and better Schrödinger cats, Phys. Rev. A 62, 013607 (2000).
[62] S. Kohler and F. Sols, Phase-resolution limit in the macroscopic interference between Bose-Einstein condensates, Phys. Rev. A 63, 053605 (2001).

[63] A. Polkovnikov, Evolution of the macroscopically entangled states in optical lattices, Phys. Rev. A 68, 033609 (2003).

[64] P. Buonsante, V. Penna, and A. Vezzani, Attractive ultracold bosons in a necklace optical lattice, Phys. Rev. A 72, 043620 (2005).

[65] J. A. Dunningham, K. Burnett, R. Roth, and W. D. Phillips, Creation of macroscopic superposition states from arrays of Bose-Einstein condensates, New J. Phys. 8, 182 (2006).

[66] Y. P. Huang and M. G. Moore, Creation, detection, and decoherence of macroscopic quantum superposition states in double-well Bose-Einstein condensates, Phys. Rev. A 73, 023606 (2006).

[67] J. I. Korsbakken, K. B. Whaley, J. Dubois, and J. I. Cirac, Measurement-based measure of the size of macroscopic quantum superpositions, Phys. Rev. A 75, 042106 (2007).

[68] D. W. Hallwood, K. Burnett, and J. Dunningham, Macroscopic superpositions of superfluid flows, New J. Phys. 8, 180 (2006).

[69] D. W. Hallwood, K. Burnett, and J. Dunningham, The barriers to producing multiparticle superposition states in rotating Bose-Einstein condensates, J. Mod. Opt. 54, 2129 (2007).

[70] A. M. Rey, K. Burnett, I. I. Satija, and C. W. Clark, Entanglement and the Mott transition in a rotating bosonic ring lattice, Phys. Rev. A 75, 063616 (2007).

[71] A. Nunnenkamp, A. M. Rey, and K. Burnett, Generation of macroscopic superposition states in ring superlattices, Phys. Rev. A 77, 023622 (2008).

[72] L. D. Carr, D. R. Dounas-Frazer, and M. A. Garcia-March, Dynamical realization of macroscopic superposition states of cold bosons in a tilted double well, Europhys. Lett. 90, 10005 (2010)

[73] D. W. Hallwood, T. Ernst, and J. Brand, Robust mesoscopic superposition of strongly correlated ultracold atoms, Phys. Rev. A 82, 063623 (2010).

[74] T. J. Haigh, A. J. Ferris, and M. K. Olsen, Demonstrating mesoscopic superpositions in double-well Bose-Einstein condensates, Opt. Commun. 283, 3540 (2010).

[75] P. Bader and U. R. Fischer, Fragmented Many-Body Ground States for Scalar Bosons in a Single Trap, Phys. Rev. Lett. 103, 060402 (2009).

[76] M.-K. Kang and U. R. Fischer, Revealing Single-Trap Condensate Fragmentation by Measuring Density-Density Correlations After Time of Flight, Phys. Rev. Lett. 113, 140404 (2014).

[77] U. R. Fischer and M.-K. Kang, 'Photonic' Cat States from Strongly Interacting Matter Waves, Phys. Rev. Lett. 115, 260404 (2015).

[78] T.-L. Ho and S. K. Yip, Fragmented and Single Condensate Ground States of Spin-1 Bose Gas, Phys. Rev. Lett. 84, 4031 (2000).

[79] B. Dakić and M. Radonjić, Macroscopic Superpositions as Quantum Ground States, Phys. Rev. Lett. 119, 090401 (2017).

[80] I. E. Mazets, G. Kurizki, M. K. Oberthaler, and J. Schmiedmayer, Creation of macroscopic quantum superposition states by a measurement, Europhys. Lett. 83, 60004 (2008). 
[81] S. Glancy and H. M. de Vasconcelos, Methods for producing optical coherent state superpositions, J. Opt. Soc. Am. 25, 712 (2008).

[82] U. R. Fischer and B. Xiong, Robustness of fragmented condensate many-body states for continuous distribution amplitudes in fock space, Phys. Rev. A 88, 053602 (2013).

[83] G. Sierra, J. Dukelsky, G. G. Dussel, J. von Delft, and F. Braun, Exact study of the effect of level statistics in ultrasmall superconducting grains, Phys. Rev. B 61, R11890 (2000).

[84] L. Amico, G. Falci, and R. Fazio, The BCS model and the off-shell Bethe ansatz for vertex models, J. Phys. A 34, 6425 (2001).

[85] F. Sols, M. Macucci, U. Ravaioli, and K. Hess, Theory for a quantum modulated transistor, J. Appl. Phys. 66, 3892 (1989).

[86] M. A. Cazalilla, R. Citro, T. Giamarchi, E. Orignac, and M. Rigol, One dimensional bosons: From condensed matter systems to ultracold gases, Rev. Mod. Phys. 83, 1405 (2011).

[87] A. L. Gaunt, T. F. Schmidutz, I. Gotlibovych, R. P. Smith, and Z. Hadzibabic, Bose-Einstein Condensation of Atoms in a Uniform Potential, Phys. Rev. Lett. 110, 200406 (2013).

[88] M. J. Everitt, T. P. Spiller, G. J. Milburn, R. D. Wilson, and A. M. Zagoskin, Engineering dissipative channels for realizing Schrödinger cats in SQUIDs, Frontiers in ICT 1, 1 (2014).

[89] H. Jeong, M. Kang, and H. Kwon, Characterizations and quantifications of macroscopic quantumness and its implementations using optical fields, Opt. Commun. 337, 12 (2015).

[90] F. Fröwis, N. Sangouard, and N. Gisin, Linking measures for macroscopic quantum states via photon-spin mapping, Opt. Commun. 337, 2 (2015).

[91] W. Dür, C. Simon, and J. I. Cirac, Effective Size of Certain Macroscopic Quantum Superpositions, Phys. Rev. Lett. 89, 210402 (2002).

[92] F. Marquardt, B. Abel, and J. von Delft, Measuring the size of a quantum superposition of many-body states, Phys. Rev. A 78, 012109 (2008).
[93] C.-W. Lee and H. Jeong, Quantification of Macroscopic Quantum Superpositions within Phase Space, Phys. Rev. Lett. 106, 220401 (2011).

[94] F. Fröwis and W. Dür, Measures of macroscopicity for quantum spin systems, New J. Phys. 14, 093039 (2012).

[95] T.-L. Ho and C. V. Ciobanu, The Schrödinger cat family in attractive Bose gases, J. Low Temp. Phys. 135, 257 (2004).

[96] A. J. Leggett, Macroscopic quantum systems and the quantum theory of measurement, Prog. Theor. Phys. Supp. 69, 80 (1980).

[97] G. Mazzarella, L. Salasnich, A. Parola, and F. Toigo, Coherence and entanglement in the ground state of a bosonic Josephson junction: From macroscopic Schrödinger cat states to separable Fock states, Phys. Rev. A 83, 053607 (2011).

[98] E. G. Cavalcanti and M. D. Reid, Signatures for Generalized Macroscopic Superpositions, Phys. Rev. Lett. 97, 170405 (2006).

[99] G. Björk and P. G. L. Mana, A size criterion for macroscopic superposition states, J. Opt. B 6, 429 (2004).

[100] J. I. Korsbakken, F. K. Wilhelm, and K. B. Whaley, Electronic structure of superposition states in flux qubits, Phys. Scr. 2009, 014022 (2009).

[101] F. Fröwis, M. Van den Nest, and W. Dür, Certifiability criterion for large-scale quantum systems, New J. Phys. 15, 113011 (2013).

[102] P. Sekatski, N. Sangouard, and N. Gisin, Size of quantum superpositions as measured with classical detectors, Phys. Rev. A 89, 012116 (2014).

[103] C. A. Fuchs and J. Van De Graaf, Cryptographic distinguishability measures for quantum-mechanical states, IEEE Trans. Inf. Theory 45, 1216 (1999).

[104] C. E. Creffield and F. Sols, Controlled Generation of Coherent Matter Currents using a Periodic Driving Field, Phys. Rev. Lett. 100, 250402 (2008). 